\title{
Japanese Classification of Esophageal Cancer, 11th Edition: part I
}

\author{
Japan Esophageal Society ${ }^{1}$
}

Published online: 10 November 2016

(c) The Author(s) 2016. This article is published with open access at Springerlink.com

President

Hisahiro Matsubara

Chiba University

Former President

Nobutoshi Ando

Tokyo Dental University

English Edition Committee, Chairman

Hisahiro Matsubara

Chiba University

English Edition Committee Members

Kenji Nemoto

Naohisa Yahagi

Soji Ozawa

Yoshiaki Kajiyama

Tatsuyuki Kawano

Tomio Arai

Yuji Tachimori

Shoji Natsugoe

Kumiko Momma

Yasuyuki Seto

Yuichiro Doki

English Edition Supervisor

Hiromasa Fujita

Yamagata University

Keio University

Tokai University

Juntendo University

Tokyo Medical and Dental University

Tokyo Metropolitan Geriatric Hospital and Institute of Gerontology

National Cancer Center Hospital

Kagoshima University

Tokyo Metropolitan Cancer and Infectious Diseases Center Komagome Hospital

Tokyo University

Osaka University

Fukuoka Wajiro Hospital

Editorial Assistants

Yasunori Akutsu

Chiba University

Japanese Edition Committee, Chairman

Hisahiro Matsubara

Chiba University

\footnotetext{
Japan Esophageal Society

office@esophagus.jp

1 Hirose-Building 4F, Taihei 2-3-13, Sumida-ku, Tokyo 130-0012, Japan
} 
Japanese Edition Committee Members

Kenji Nemoto

Naohisa Yahagi

Soji Ozawa

Yoshiaki Kajiyama

Tatsuyuki Kawano

Tomio Arai

Yuji Tachimori

Shoji Natsugoe

Kumiko Momma

Yasuyuki Seto

Yuichiro Doki
Yamagata University

Keio University

Tokai University

Juntendo University

Tokyo Medical and Dental University

Tokyo Metropolitan Geriatric Hospital and Institute of Gerontology

National Cancer Center Hospital

Kagoshima University

Tokyo Metropolitan Cancer and Infectious Diseases Center Komagome Hospital

Tokyo University

Osaka University

Pathological Research Committee, Chairman

Tomio Arai

Tokyo Metropolitan Geriatric Hospital and Institute of Gerontology

Pathological Research Committee Members

Yasuo Ohkura

Shingo Ishiguro

Hiroshi Kawachi

Kaiyo Takubo

Masamitsu Unakami

Takashi Yao

Suguru Yonezawa

Tetsuo Nemoto

Endoscopy Research Committee, Chairman

Tuneo Oyama

Saku Central Hospital

Endoscopy Research Committee Members

Kumiko Momma

Tai Omori

Tatsuyuki Kawano

Hideo Shimada

Manabu Takeuchi

Ken Haruma

Ryu Ishihara

Akio Yanagisawa

Ryoji Kushima

Kyorin University
PCL Japan

The Cancer Institute Hospital of Japanese Foundation for Cancer Research

Tokyo Metropolitan Institute of Gerontology

Watari Hospital

Juntendo University

Kagoshima University

Toho University
Tokyo Metropolitan Cancer and Infectious Diseases Center Komagome Hospital Kawasaki Municipal Ida Hospital

Tokyo Medical and Dental University

Tokai University Oiso Hospital

Nagaoka Red Cross Hospital

Kawasaki Medical School

Osaka Medical Center for Cancer and Cardiovascular Diseases

Kyoto Prefectural University of Medicine

Shiga University of Medical Science 


\section{Contents}

Preface

General principles of this edition

Abbreviations

Part I General rules 10

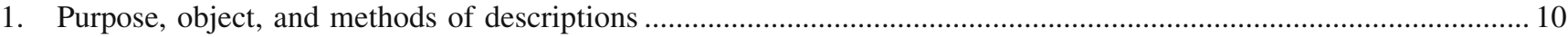

1.1. Purpose

1.2. Object ...

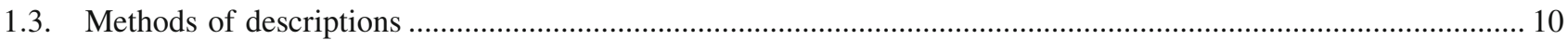

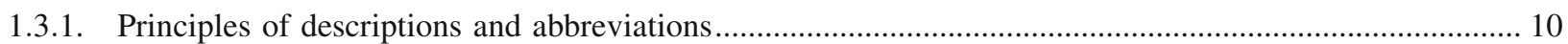

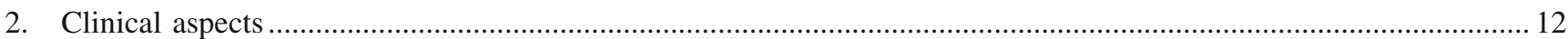

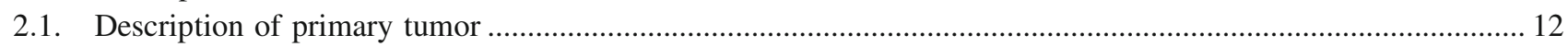

2.1.1. Number of primary tumors, size and circumferential location ............................................................. 12

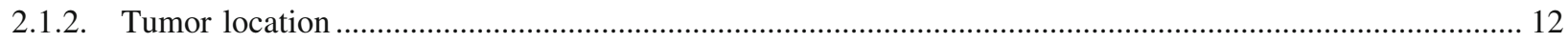

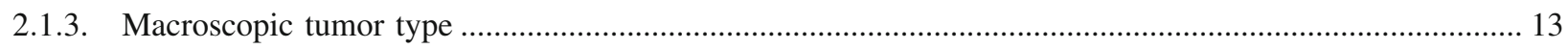

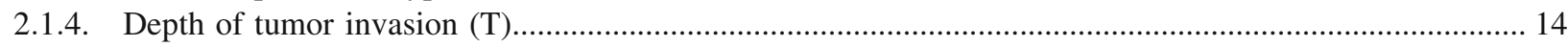

2.2. Metastatic lesions from esophageal cancerMetastatic lesions from esophageal cancer ....................................... 15

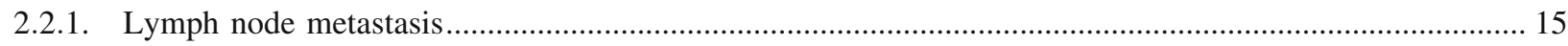

2.2.2. Distant organ metastasis (M)

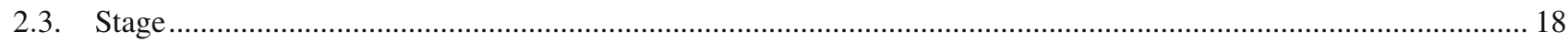

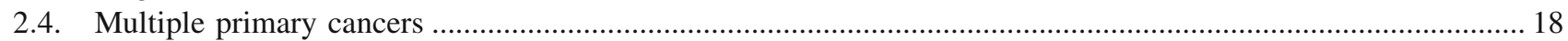

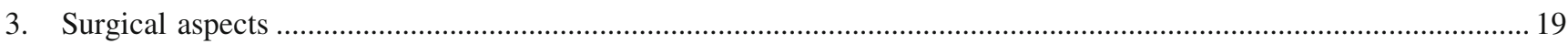

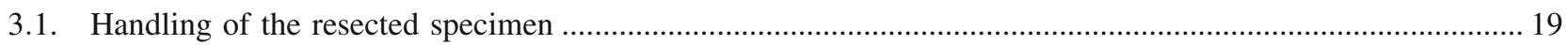

3.2. Description of surgical findings and macroscopic findings of primary tumor ................................................. 19

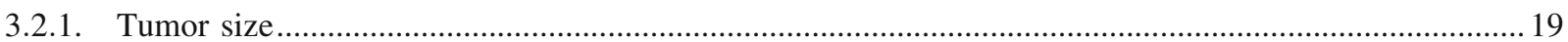

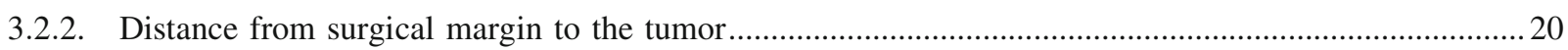

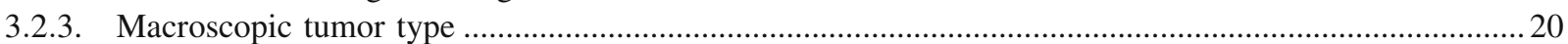

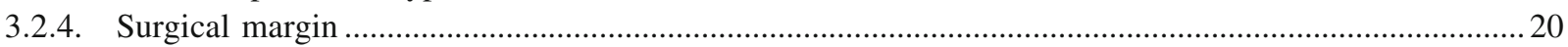

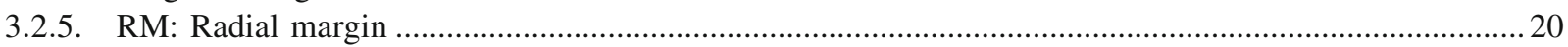

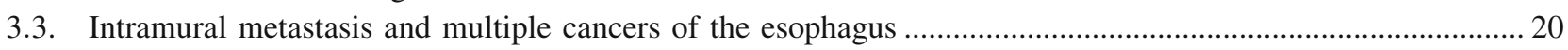

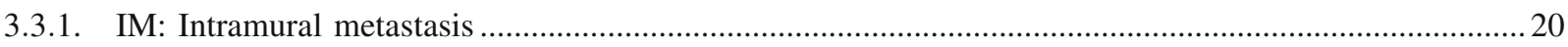

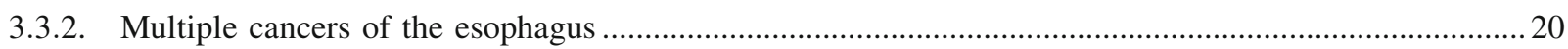

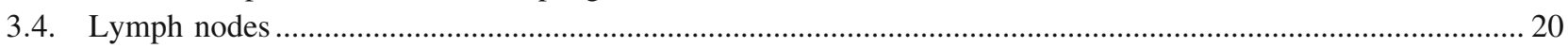

3.4.1. Preparation of resected lymph nodes for pathological examination .....................................................2 20

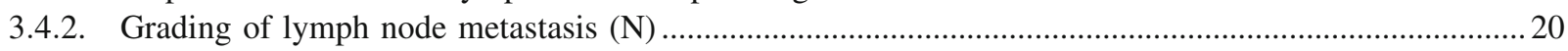

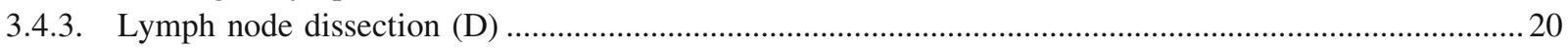

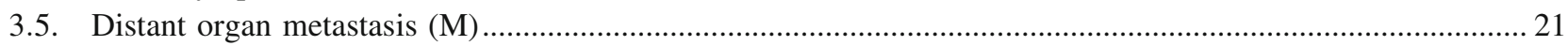

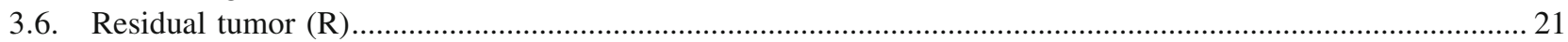

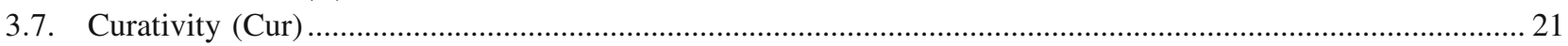

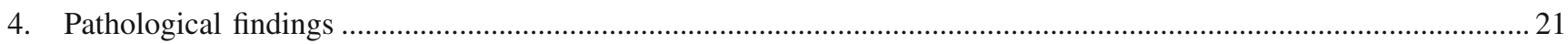

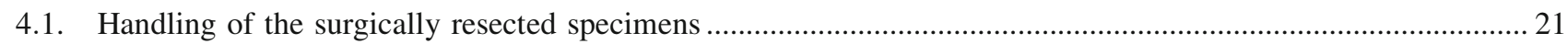

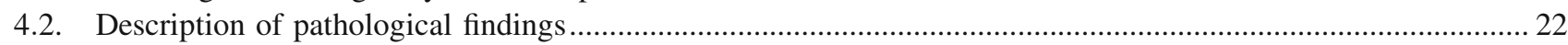

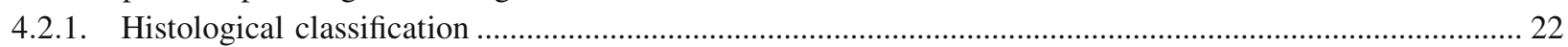

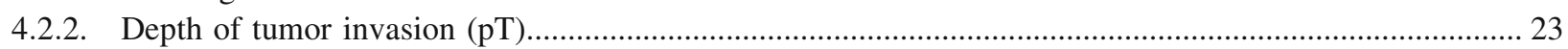

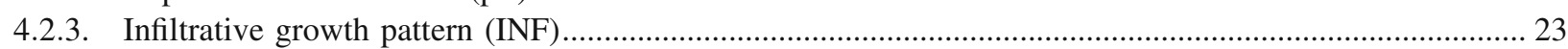

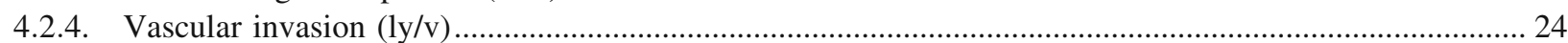

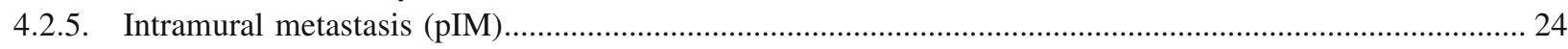

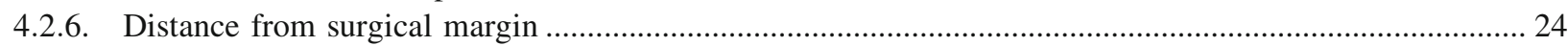




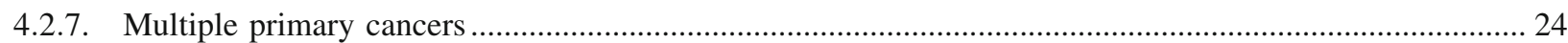

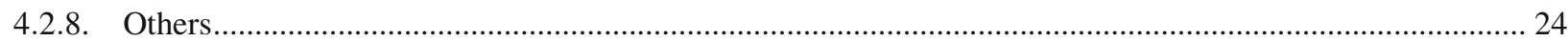

4.2.9. Pathological criteria for the effects of radiation and/or chemotherapy .................................................. 24

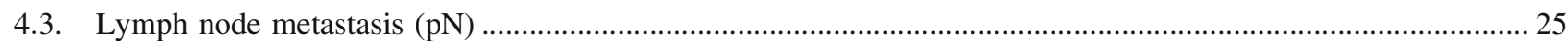

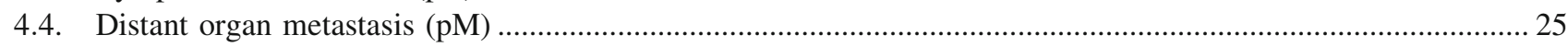

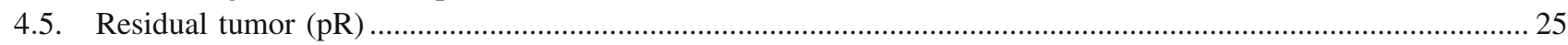

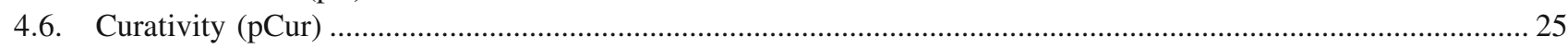

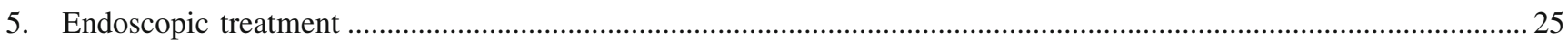

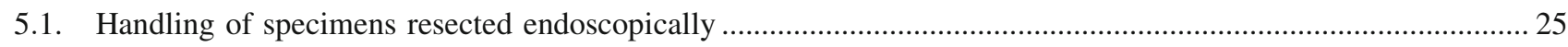

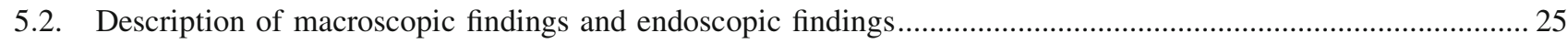

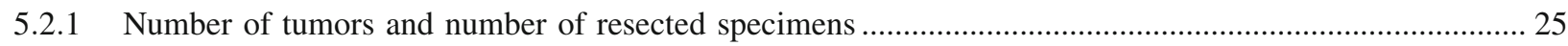

5.2.2 Size of resected specimen and size of tumor lesion (for each lesion) .................................................. 25

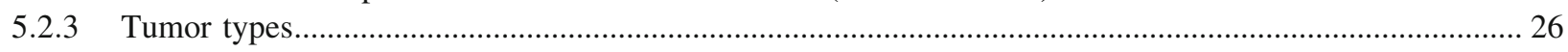

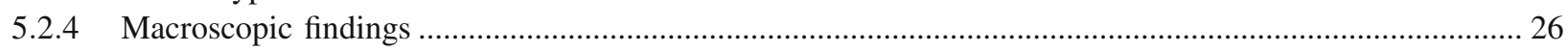

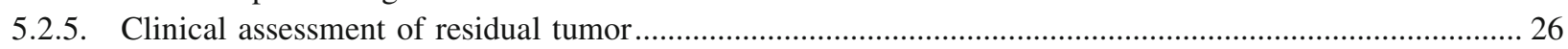

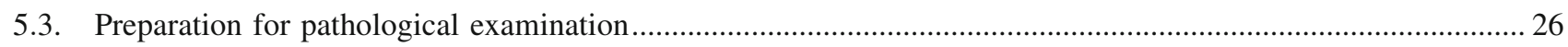

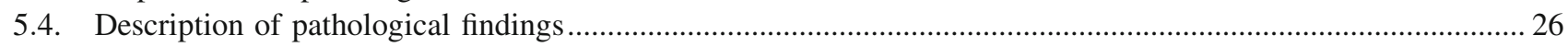

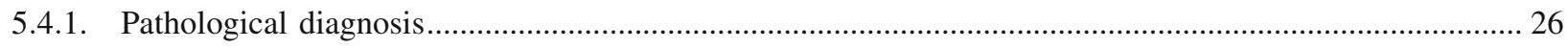

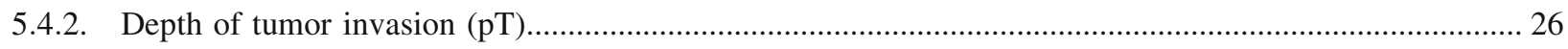

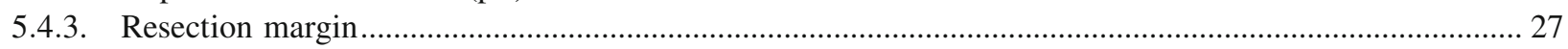

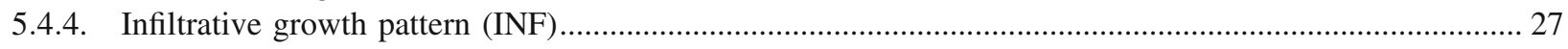

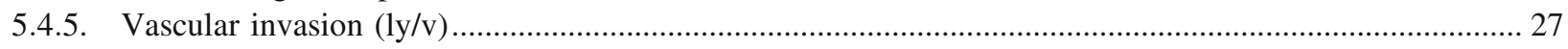

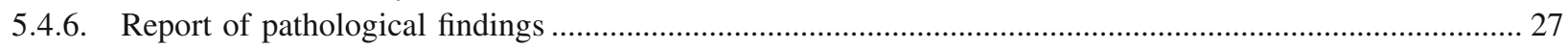

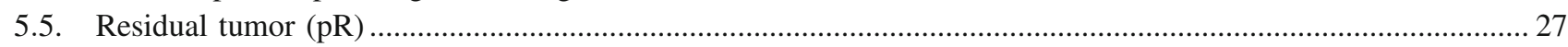

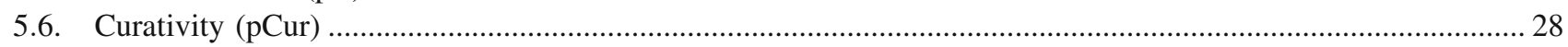

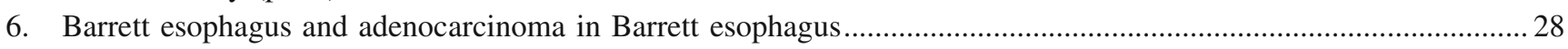

6.1. Definition and description methods for Barrett mucosa, Barrett esophagus and adenocarcinoma

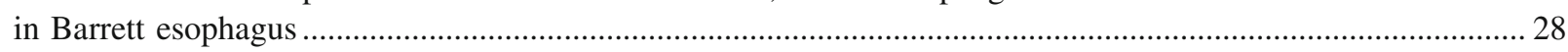

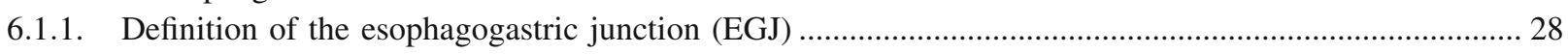

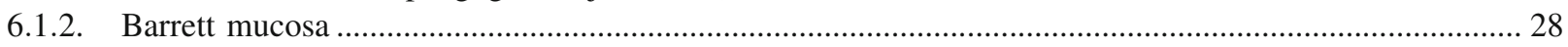

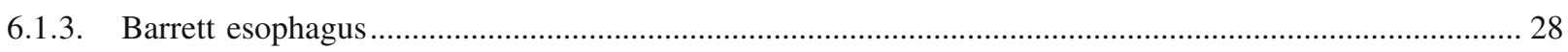

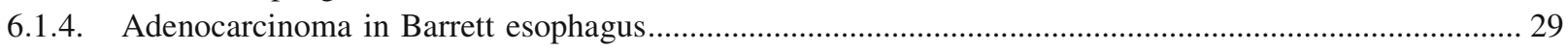

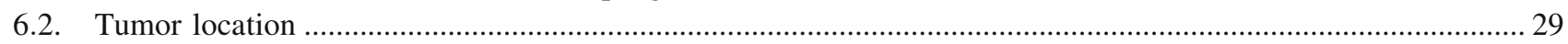

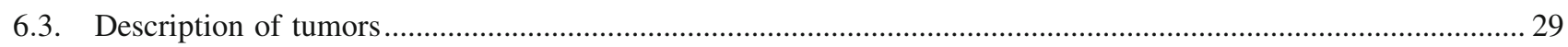

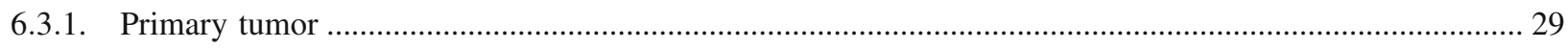

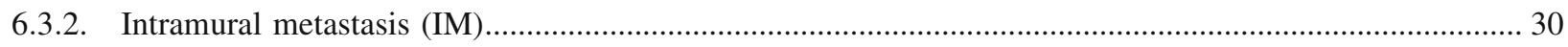

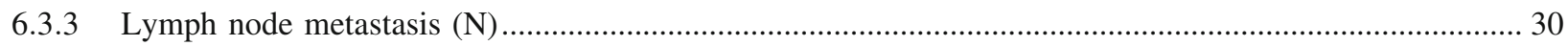

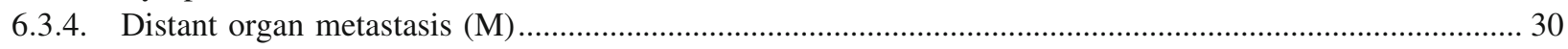

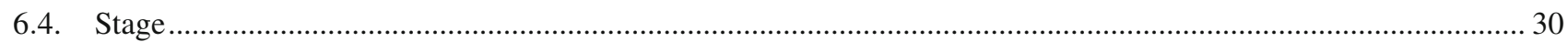

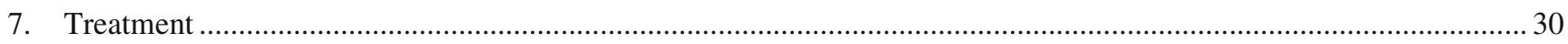

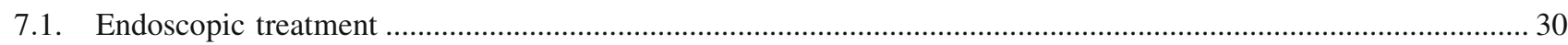

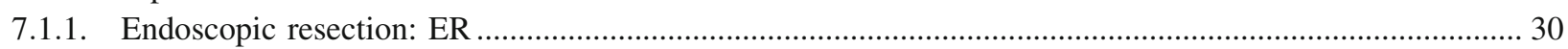

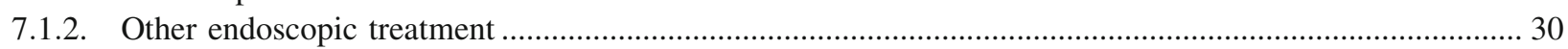

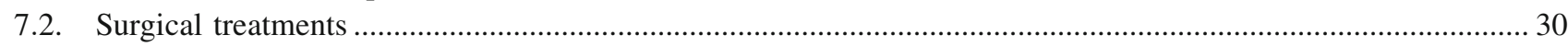

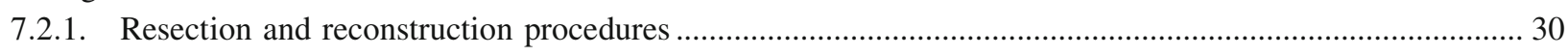

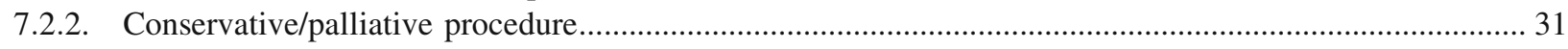

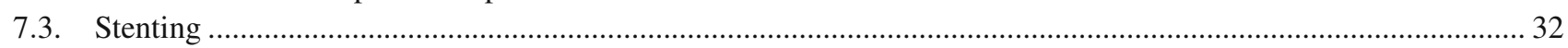

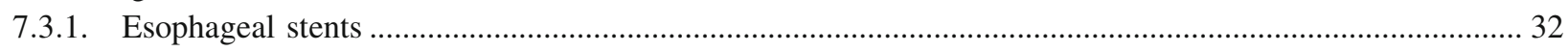

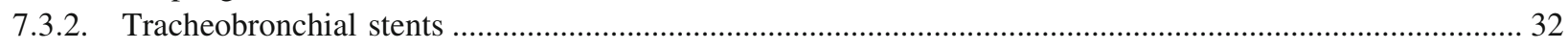

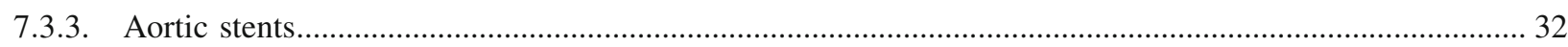




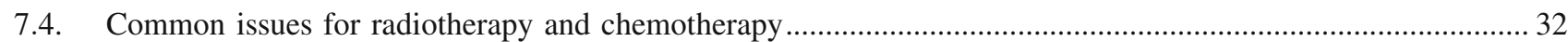

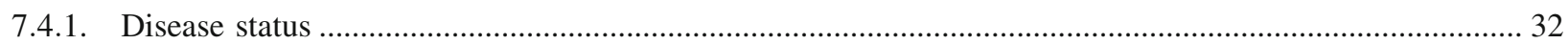

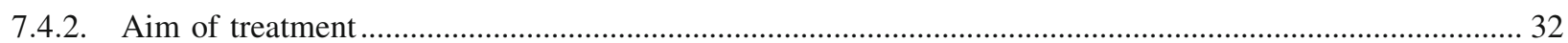

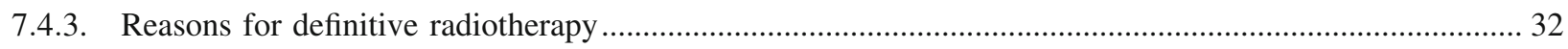

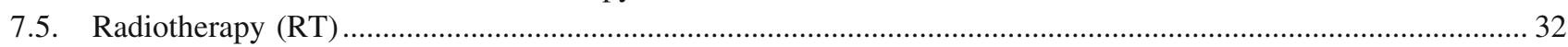

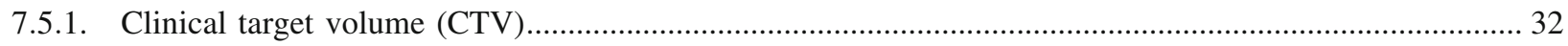

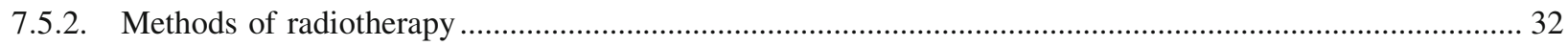

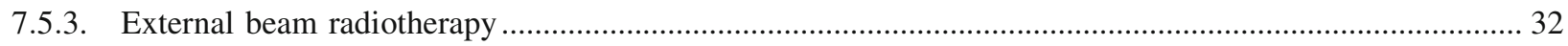

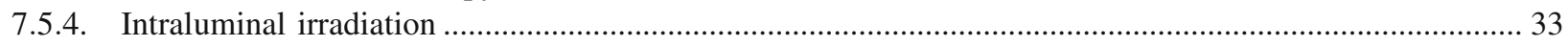

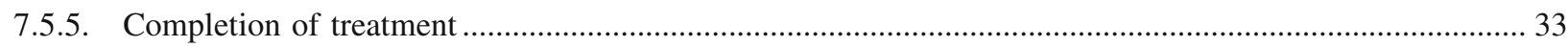

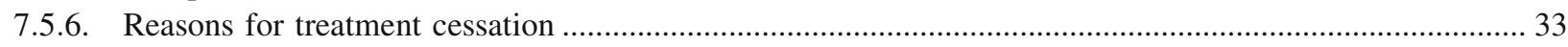

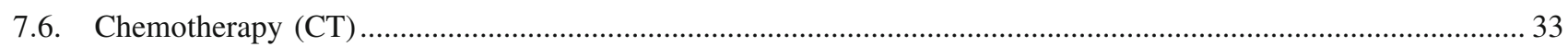

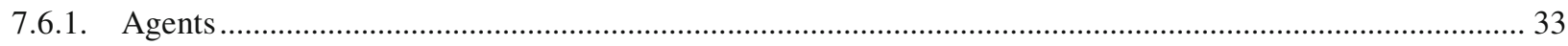

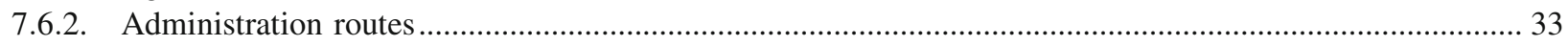

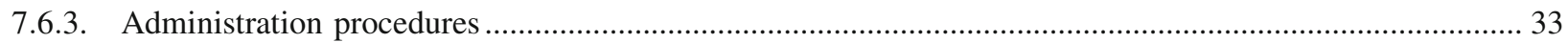

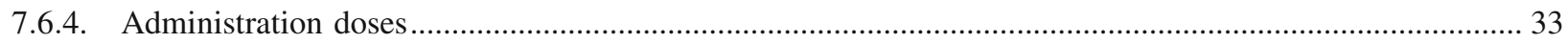

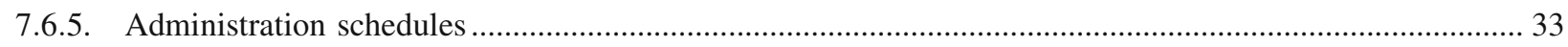

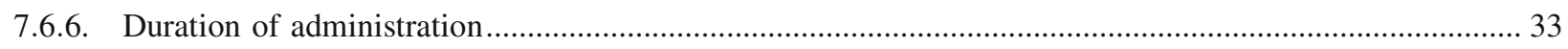

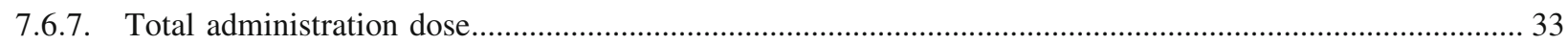

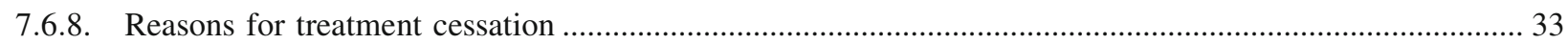

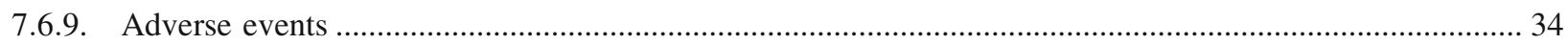

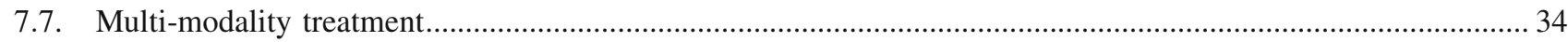

7.7.1. Combination of endoscopic treatment and surgery, radiotherapy, chemoradiotherapy or chemotherapy.. 34

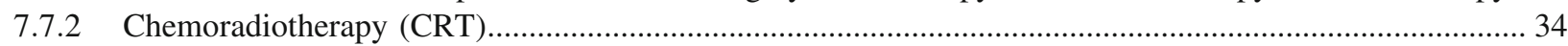

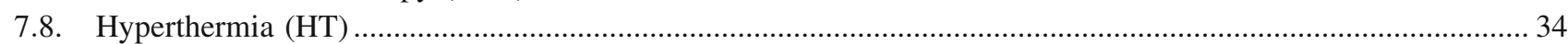

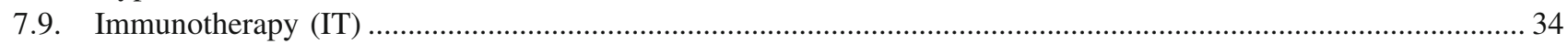

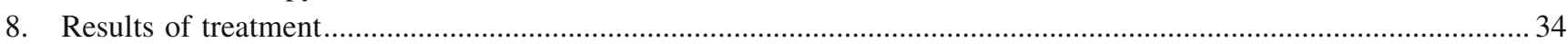

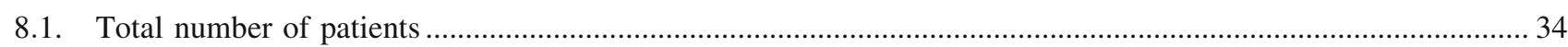

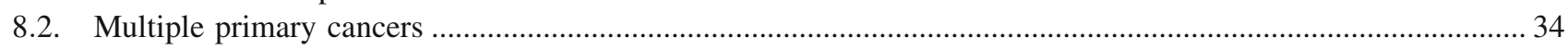

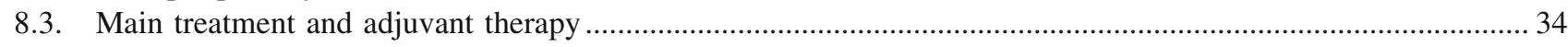

8.4. Total number of patients treated, and number and ratio of patients treated with each procedure …….............. 34

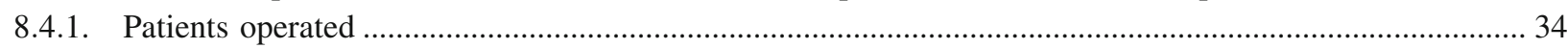

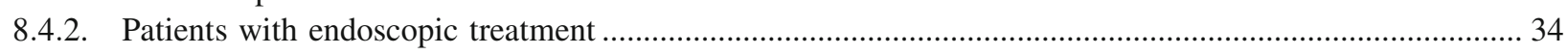

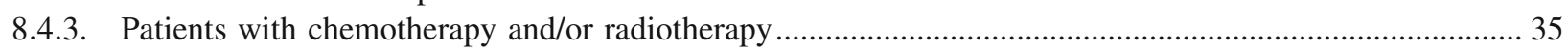

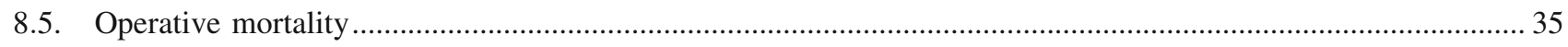

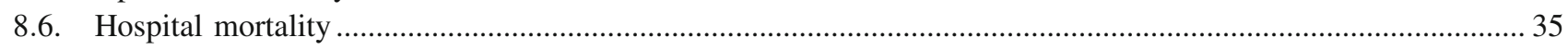

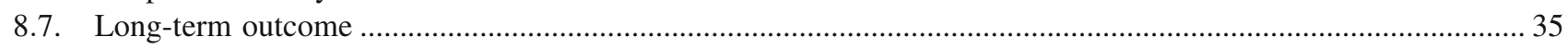

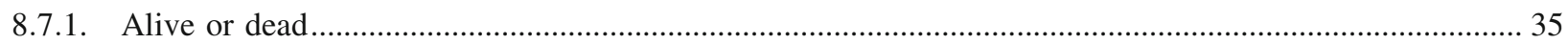

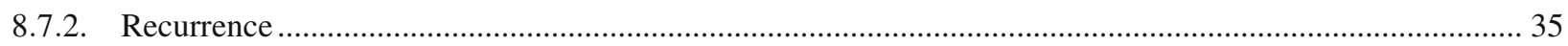

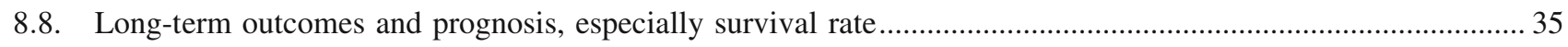

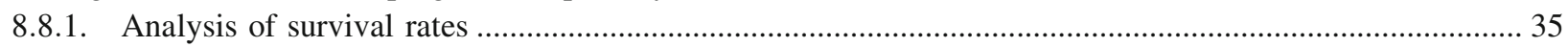

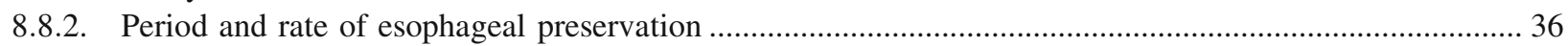

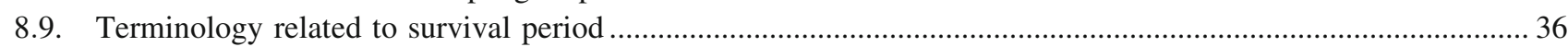

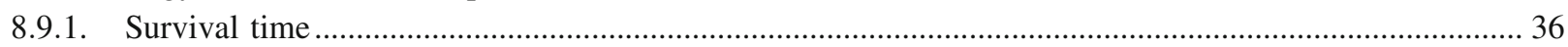

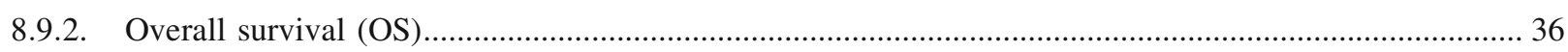

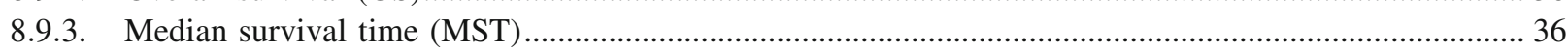

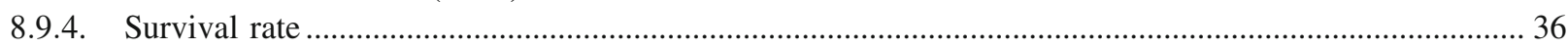

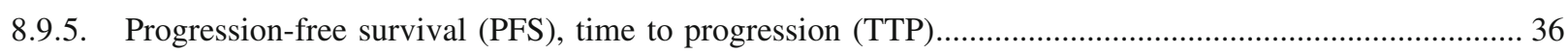

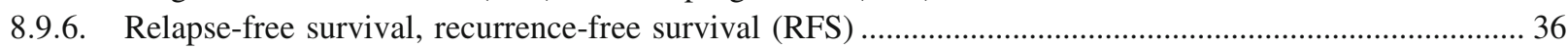




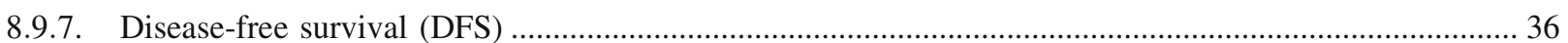

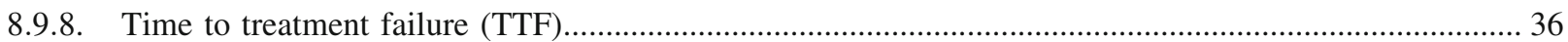

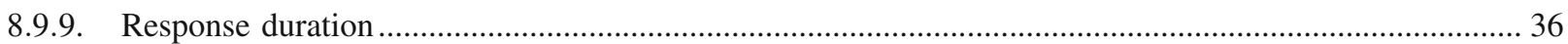

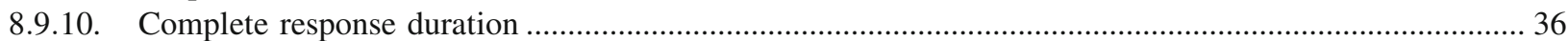




\section{Preface to the 11th Edition}

Eight years after the publication of the 10th edition in 2007, the 11th edition of the Japanese Classification of Esophageal Cancer has now been published. During this period, supplements to the 10th edition involving the revision of "disease typing" and terminology were published in 2008; in addition, following the adoption of criteria for the diagnosis of lesions located at the gastroesophageal junction that was made in cooperation with the Japanese Gastric Cancer Association, a 7-page leaflet was attached to this Classification in September 2013. The present revision was aimed at ensuring consistency with other general rules for surgical and pathological studies on cancer as far as possible, reflecting the latest advances in the diagnosis and treatment of esophageal cancer in Japan and providing a set of rules that are easier to use and that facilitate improvements in treatment outcomes. During this revision, we attempted to secure consistency with the UICC's TNM classification as far as possible. However, this attempt was skipped for the $\mathrm{N}$ classification, since the current edition (7th) of the TNM classification does not reflect the nationwide registry data of the Japan Esophageal Society and because the rules for studies on supraclavicular lymph nodes are completely different between our classification and the $\mathrm{N}$ classification. This is a significant issue that will need to be addressed in the next revision.

Using nationwide registry data, the effects of regional lymph node excision were reviewed from the viewpoints of lymph node metastasis and the survival rate. As a result, the lymph node groupings were modified (T4 was subdivided into two subtypes, similar to the TNM classification). Following recent advances and the spread of endoscopic treatment, findings from endoscopic treatment have now been incorporated into the description methods, and the exclusion of cancer from intraepithelial neoplasms has been clarified. This revised edition has been prepared as a result of numerous discussions among committee members. Although there are still some questions to be discussed, we wish to take this opportunity to thank the considerable efforts made by the individual committee members.

October, 2015 


\section{General principles of this edition}

1. Following the spread of endoscopic treatment, findings from endoscopic treatment (e) have been added to the methods used to describe findings.

2. The criteria for the diagnosis of lesions located at the gastroesophageal junction, which have been jointly adopted by the Japanese Gastric Cancer Association, have been added to the main text.

3. Regarding the depth of tumor invasion, the subgroup $\mathrm{T} 1 \mathrm{~b}$ - has been added to the subgroup T1b, similar to that for T1a, and the subgroup T4 has been further subdivided into T4a and T4b so as to be consistent with the UICC's TNM classification.

4. Regarding lymph nodes, No. 112ao has been divided into the esophageal side and the dorsal side. Furthermore, to secure consistency with the general rules for surgical and pathological studies on gastric cancer, No. 3 has been divided into No. 3a and No. $3 \mathrm{~b}$.

5. Regarding lymph node grouping, modifications have been made to Ut (Group 3 only), Mt/Lt (Groups 1, 2, 3), and Ae (Groups 2, 3). In accordance with the revision of the criteria for the diagnosis of lesions located at the gastroesophageal junction, the same lymph node classification as that used for Ae will now be applied to cancer of the gastroesophageal junction.

6. Regarding the stage of cancer, T1aN1 is now classified as Stage II, as is the case with T1bN1. T4a up to N3 is now classified as Stage III. T4b, beginning with N0, is now classified as Stage IVa.

7. Regarding the extent of residual cancer, classification into R1 based on macroscopic findings is now avoided, consistent with the general rules for surgical and pathological studies on colorectal cancer.

8. Regarding histopathological findings, it has now been made clear that carcinoma in situ is not to be included among neoplasms within the squamous epithelium. The extent of differentiation of both squamous cell carcinoma and adenocarcinoma is now described as "well differentiated" or a similar expression, omitting any description of type. Endocrine cell neoplasm is now called neuroendocrine tumor, consistent with the WHO classification. Also, concerning extralymph node metastasis, the expression "tumor nodule" has been adopted, consistent with the general rules for surgical and pathological studies on colorectal cancer. Vascular invasion in specimens collected during endoscopic treatment is now rated as $(-)$ or $(+)$, consistent with the method used for gastric cancer.

9. The TNM classification adopted for the revised classification has been switched to the Japanese translation of the TNM classification, 7th edition.

10. Regarding the number of lymph node metastases, the conventional rule for the correction of grouping according to the number of metastases was too complex and was not used frequently. This rule has been deleted from the revised edition.

11. The endoscope pictures have been replaced with clearer ones.

12. Regarding the extents and borders of the lymph nodes, not only schematic figures, but also actual CT images have been provided to simplify understanding, accompanied by the presentation of features that will also be useful for radiotherapy. 


\section{Abbreviations}

$\mathrm{AD}$

$\mathrm{Ae} \quad$ Abdominal esophagus

AI Invasion to the adjacent structures

APC Argon plasma coagulation

B Tracheal bifurcation

c Clinical findings

$\mathrm{Ce} \quad$ Cervical esophagus

CR Complete response

CRT Chemoradiotherapy

CT Chemotherapy

CTV Clinical target volume

Cur Curativity

D Lymph node dissection

DFS Disease-free survival

DM Distal margin

DMM Deep muscularis mucosae

E Esophagus

EG Tumor located in the esophageal side

EGJ Esophagogastric junction

EMR Endoscopic mucosal resection

EP Epithelium [p.41, 74]

ER Endoscopic resection

ESD Endoscopic submucosal dissection

EVG Elastica van Gieson staining

f Final findings

G Stomach

GE Tumor located in the gastric side

GIST Gastrointestinal stromal tumor

$\mathrm{H} \quad$ Esophageal hiatus

HM Horizontal margin

HT Hyperthermia

IM Intramural metastasis

INF Infiltrative growth pattern

IR/SD Incomplete response/stable disease

IT Immunotherapy

LN Lymph node

LPM Lamina propria mucosae

LSBE Long segment Barrett esophagus

Laser Laser therapy

Lt Lower thoracic esophagus

Ly Lymphatic invasion

ly/v Lymphatic invasion or venous invasion

M Distant organ metastasis

MCT Microwave coagulation therapy

MFH Malignant fibrous histiocytoma

MM Muscularis mucosae

MP Muscularis propria

MST Median survival time

Mt Middle thoracic esophagus

$\mathrm{N} \quad$ Lymph node metastasis

O Esophageal orifice

$\begin{array}{ll}\text { OS } & \text { Overall survival } \\ \text { p } & \text { Pathological findings } \\ \text { PD } & \text { Progressive disease } \\ \text { PDT } & \text { Photodynamic therapy } \\ \text { PFS } & \text { Progression-free survival } \\ \text { Ph } & \text { Pharynx } \\ \text { PM } & \text { Proximal margin } \\ \text { PR } & \text { Partial response } \\ \text { R } & \text { Residual tumor } \\ \text { RECIST } & \text { Response Evaluation Criteria in Solid Tumors } \\ \text { RFS } & \text { Relapse/recurrence-free survival } \\ \text { RM } & \text { Radial margin } \\ \text { RT } & \text { Radiotherapy } \\ \text { s } & \text { Surgical findings } \\ \text { S } & \text { Superior margin of the sternum } \\ \text { SCE } & \text { Specialized columnar epithelium } \\ \text { SCJ } & \text { Squamocolumnar junction } \\ \text { SD } & \text { Stable disease } \\ \text { SM } & \text { Submucosa } \\ \text { SMM } & \text { Superficial muscularis mucosae } \\ \text { SSBE } & \text { Short segment Barrett esophagus } \\ \text { T } & \text { Depth of tumor invasion } \\ \text { Te } & \text { Thoracic esophagus } \\ \text { Tis } & \text { Carcinoma in situ } \\ \text { TT } & \text { Thermotherapy } \\ \text { TTF } & \text { Time to treatment failure } \\ \text { TTP } & \text { Time to progression } \\ \text { Ut } & \text { Upper thoracic esophagus } \\ \text { v } & \text { Venous invasion } \\ \text { VB } & \text { Victoria blue staining } \\ \text { VM } & \text { Vertical margin [p. 39] } \\ \text { X } & \text { Cannot be assessed [p. 2] } \\ & \end{array}$

\section{Terminology of the lymph nodes}

$\begin{array}{ll}\text { R } & \text { Right } \\ \text { L } & \text { Left } \\ \text { sm } & \text { Submandibular } \\ \text { spf } & \text { Superficial } \\ \text { ac } & \text { Accessory } \\ \text { tr } & \text { Tracheal } \\ \text { up } & \text { Upper } \\ \text { mid } & \text { Middle } \\ \text { rec } & \text { Recurrent nerve } \\ \text { tb } & \text { Tracheobronchial } \\ \text { pre } & \text { Pretracheal } \\ \text { ao } & \text { Paraaortic } \\ \text { pul } & \text { Pulmonary ligament }\end{array}$

Number of the lymph nodes

a: $1-3$, b: $4-7$, c: $\geqq 8$ 


\section{Part I \\ General rules}

\section{Purpose, object, and methods of descriptions}

\subsection{Purpose}

"The Guidelines for the Clinical and Pathologic Studies on Carcinoma of the Esophagus" was originally published in 1969 by the Japanese Society for Esophageal Diseases. Since then, the Society has changed its name in 2003 to become the Japan Esophageal Society, and has published the "Japanese Classification of Esophageal Cancer" in Japanese with some revisions to keep up to date with treatment results and to provide a standard nomenclature. To promote the international use of the Guidelines and the Classification, the Society is publishing this handbook in English entitled "The Japanese Classification of Esophageal Cancer".

\subsection{Object}

The term esophageal cancer in the Japanese Classification refers to cancer originating in the esophagus, and cancer metastatic to the esophagus is excluded. All primary malignant tumors in the esophagus should be described according to the Japanese Classification.

\subsection{Methods of descriptions}

\subsubsection{Principles of descriptions and abbreviations}

Findings are recorded using upper-case letters $\mathrm{T}$ (depth of tumor invasion), $\mathrm{N}$ (lymph node metastasis) and $\mathrm{M}$ (distant organ metastasis). The extent of each finding is expressed by Arabic numerals following each uppercase letter. " $\mathrm{X}$ " is used in unknown cases. Five categories of findings, namely Clinical, Endoscopic treatment, Surgical, Pathological, and Final findings, are identified using the lower case "c", "e", "s", "p", and "f", respectively, before each upper-case letter. The " $\mathrm{f}$ " of Final findings can be omitted (Tables 1-1, 1-2). Checklists for descriptions of the Japanese Classification of Esophageal Cancer are shown in the following tables (Tables 1-3, 1-4).

The order of clinicopathological description is:

Tumor location (in addition to describing the distance from the incisor), circumferential extent, tumor length, macroscopic tumor type, histological type (when identified), depth of tumor invasion, lymph node metastasis, distant organ metastasis and stage.

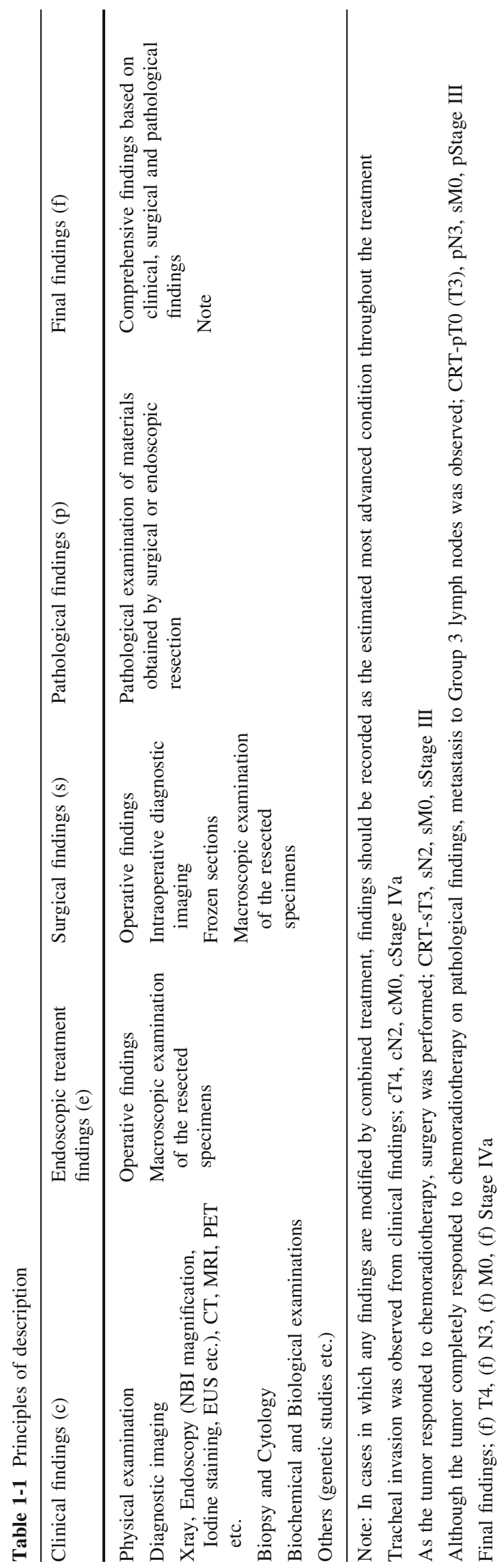


Table 1-2 Description methods

\begin{tabular}{|c|c|c|c|c|c|}
\hline & Clinical findings & Endoscopic treatment findings & Surgical findings & Pathological findings & Final findings \\
\hline Depth of tumor invasion & cT & $\mathrm{eT}$ & sT & pT & (f) $\mathrm{T}$ \\
\hline Lymph node metastasis & $\mathrm{cN}$ & - & $\mathrm{sN}$ & $\mathrm{pN}$ & (f) $\mathrm{N}$ \\
\hline Distant organ metastasis & $\mathrm{cM}$ & - & $\mathrm{sM}$ & $\mathrm{pM}$ & (f) $\mathrm{M}$ \\
\hline Intramural metastasis & cIM & eIM & sIM & pIM & (f) IM \\
\hline Stage & cStage & eStage & sStage & pStage & (f) Stage \\
\hline Proximal margin & - & - & sPM & pPM & (f) $\mathrm{PM}$ \\
\hline Distal margin & - & - & sDM & $\mathrm{pDM}$ & (f) $\mathrm{DM}$ \\
\hline Radial margin & - & - & sRM & $\mathrm{pRM}$ & (f) $\mathrm{RM}$ \\
\hline Horizontal margin (EMR/ESD) & - & eHM & sHM & $\mathrm{pHM}$ & (f) $\mathrm{HM}$ \\
\hline Vertical margin (EMR/ESD) & - & eVM & sVM & $\mathrm{pVM}$ & (f) $\mathrm{VM}$ \\
\hline Residual tumor & - & eR & sR & $\mathrm{pR}$ & (f) $\mathrm{R}$ \\
\hline Curativity & - & eCur & sCur & pCur & (f) $\mathrm{Cur}$ \\
\hline
\end{tabular}

Findings modified by treatment methods other than surgery are abbreviated as follows: $R T$ radiotherapy, $C T$ chemotherapy, $C R T$ chemoradiotherapy, EMR endoscopic mucosal resection, ESD endoscopic submucosal dissection, laser laser therapy, $P D T$ photodynamic therapy

Table 1-3 Checklist for descriptions of the Japanese Classification of Esophageal Cancer (surgically treated cases)

Tumor location: Ce, Ut, Mt, Lt, Ae

Size: maximum length $(\mathrm{mm})$ and orthogonally oriented maximum width (mm)

Macroscopic tumor type:

Type 0 , Type 1 , Type 2, Type 3, Type 4, Type 5, Combined type, Others

Subclassification for superficial cancers: 0-I(0-Ip, 0-Is), 0-II(0-IIa, 0-IIb, 0-IIc), 0-III

Histological type: squamous cell carcinoma, basaloid (-squamous) carcinoma, carcinosarcoma, adenocarcinoma, Barrett's adenocarcinoma, adenosquamous carcinoma, mucoepidermoid carcinoma, adenoid cystic carcinoma, neuroendocrine cell tumor, undifferentiated carcinoma, other carcinomas, non-epithelial malignant tumors, GIST, malignant melanoma

Depth of tumor invasion: pTX, pT0, pT1a (EP, LPM, MM), pT1b (SM1, SM2, SM3), pT2, pT3, pT4

Pattern of infiltration: INFa, INFb, INFc

Lymphatic invasion: 1y0, ly1, ly2, ly3

Venous invasion: v0, v1, v2, v3

Intramural metastasis: pIMX, pIM0, pIM1

Involvement of resection margins

Proximal margin: pPMX, pPM0, pPM1

Distal margin: pDMX, pDM0, pDM1

Radial margin: pRMX, pRM0, pRM1

Multiple primary cancers: absent, present (number)

Lymph node metastasis: pNX, pN0, pN1, pN2, pN3, pN4

Number of positive nodes (No. of lymph node stations with positive nodes)

Distant metastasis: M0, M1

Residual tumor: $\mathrm{pRX}, \mathrm{pR} 0, \mathrm{pR} 1, \mathrm{pR} 2$

Histological curativity: pCurA, pCurB, pCurC

Distant organ metastasis: MX, M0, M1; Intramural gastric metastasis: pIM1-St

Therapeutic efficacy: No data, Grade 0, Grade 1a, Grade 1b, Grade 2, Grade 3

Curativity: Cur A, Cur B, Cur C 
Table 1-4 Checklist for descriptions of the Japanese Classification of Esophageal Cancer (endoscopically treated cases)

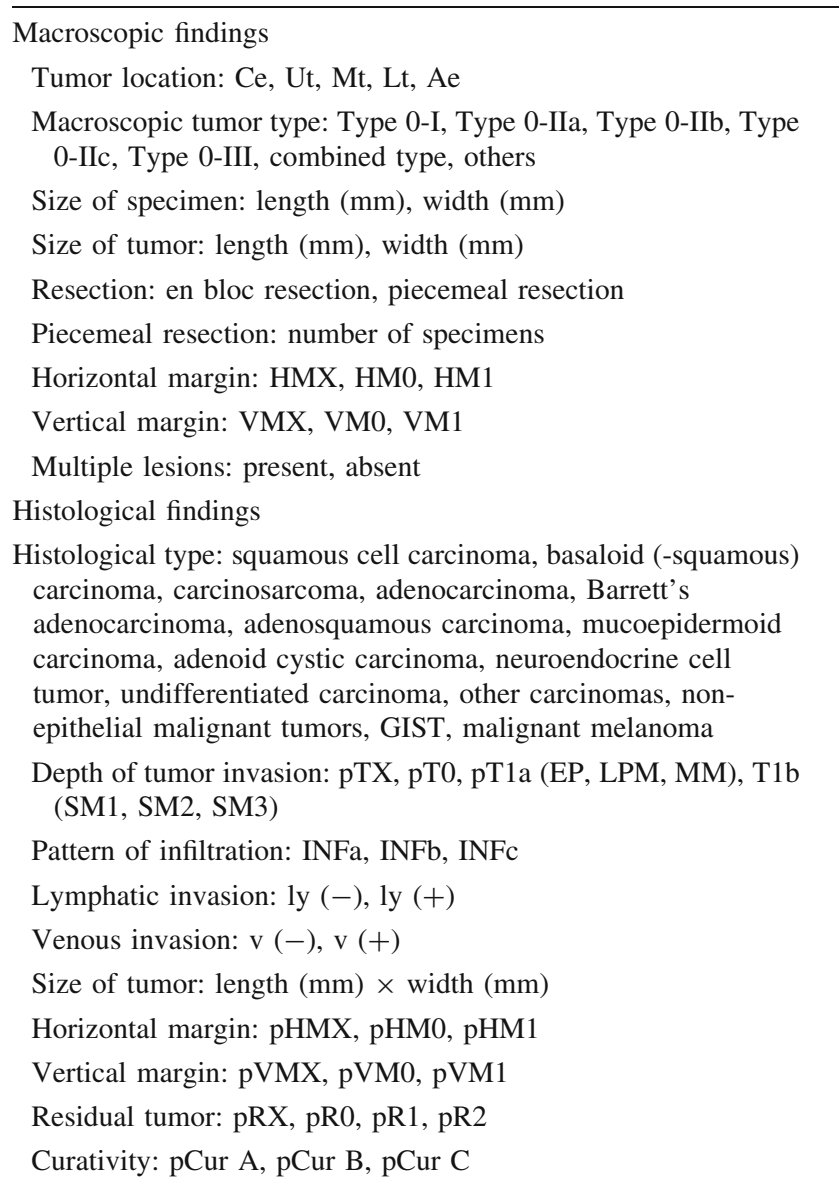

e.g.: Mt (31-36 cm), 1/2 of circumference and on anterior wall, $5 \mathrm{~cm}$, Type 2 , moderately differentiated squamous cell carcinoma, pT3, pN2, sM0, fStage III.

The order of pathological description is:

Tumor location, tumor length, macroscopic tumor type, histological type, depth of tumor invasion, pattern of infiltration, lymphatic invasion, venous invasion, intramural metastasis, involvement of the resection margins (proximal margin, distal margin, and radial margin), multiple primary cancers, effects of radiation and/or anticancer chemotherapy, lymph node metastasis, distant organ metastasis, and stage.

e.g.: Mt, $5 \mathrm{~cm}$, Type 2, moderately differentiated squamous cell carcinoma, pT3, INFa, ly1, v1, IM0, pPM0, pDM0, pRM0, multiple primary carcinomas (present, two lesions), CRT-grade 2, pN1 (2/30), sM0, fStage III.

\section{Clinical aspects}

\subsection{Description of primary tumor}

\subsubsection{Number of primary tumors, size and circumferential location}

Maximum length (mm) and orthogonally oriented maximum width $(\mathrm{mm})$, center of circumferential extent, and circumferential ratio of the tumor to the entire esophagus should be described. In addition, the methods used for diagnosis, such as barium X-ray, endoscopy and EUS, should be recorded.

\subsubsection{Tumor location}

\subsubsection{Anatomical definition of the esophagus}

The esophagus is defined anatomically from the esophageal orifice to the esophagogastric junction. The esophageal orifice is at the lower margin of the cricoid cartilage. The identification of the esophagogastric junction (EGJ) will be described later.

\subsubsection{Anatomical regions (subsites) of the esophagus}

The esophagus lies between the hypopharynx and stomach, and can be anatomically divided into the following portions; cervical esophagus (Ce), thoracic esophagus (Te) and abdominal esophagus (Ae). The zone of the esophagogastric junction is divided into the esophageal side (E) and gastric side (G) (Fig. 1-1). ${ }^{\text {Note }}$

Cervical esophagus (Ce): This extends from the esophageal orifice to the sternal notch.

Thoracic esophagus (Te): From the sternal notch to the superior margin of the esophageal hiatus.

Upper thoracic esophagus (Ut): From the sternal notch to the tracheal bifurcation.

Middle thoracic esophagus (Mt): The proximal half of the two equal portions between the tracheal bifurcation and the esophagogastric junction.

Lower thoracic esophagus (Lt): The thoracic part of the distal half of the two equal portions between the tracheal bifurcation and the esophagogastric junction.

Abdominal esophagus (Ae): The abdominal part of the distal half of the two equal portions between the tracheal bifurcation and the esophagogastric junction (from the 


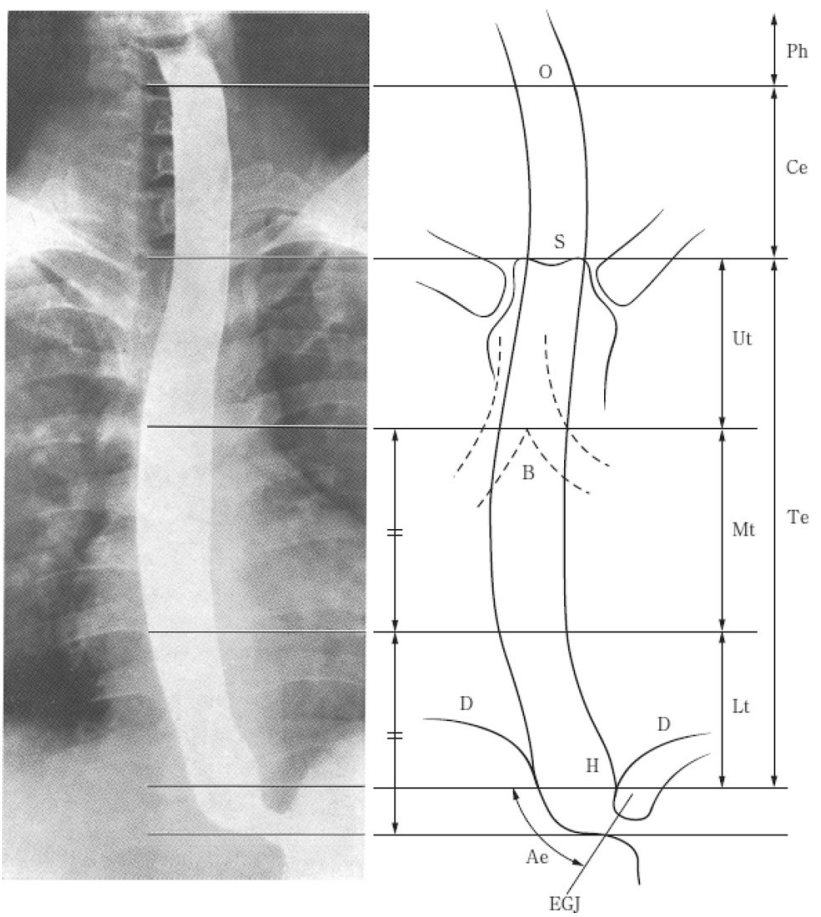

Fig. 1-1 Tumor location. $O$ esophageal orifice, $S$ superior margin of the sternum, $B$ tracheal bifurcation, $D$ diaphragm, $E G J$ esophagogastric junction, $H$ esophageal hiatus

superior margin of the esophageal hiatus to the esophagogastric junction).

Note: The zone of the esophagogastric junction is defined as the region between $2 \mathrm{~cm}$ in esophagus and $2 \mathrm{~cm}$ in the stomach from the esophagogastric junction. The abdominal esophagus is included in this zone.

\subsubsection{Principles of description of tumor location}

Describe the tumor location identified by examinations according to the following order of priority: barium X-ray, $\mathrm{CT}$, and endoscopic measurements. Include the distance from the incisor in addition to the tumor location. When the tumor location is uncertain because examinations other than endoscopy have yet to be performed, describe only the distance from the incisor.

When the tumor extends continuously in more than one portion of the esophagus, the main tumor location is that with the deepest tumor invasion. If it is difficult to determine the site of deepest tumor invasion, the portion at the central point of the tumor can be recorded as the main tumor location.

In the case of multiple primary lesions, the locations of the lesions are described in the order of depth of tumor invasion. The deepest lesion is described first. If it is difficult to

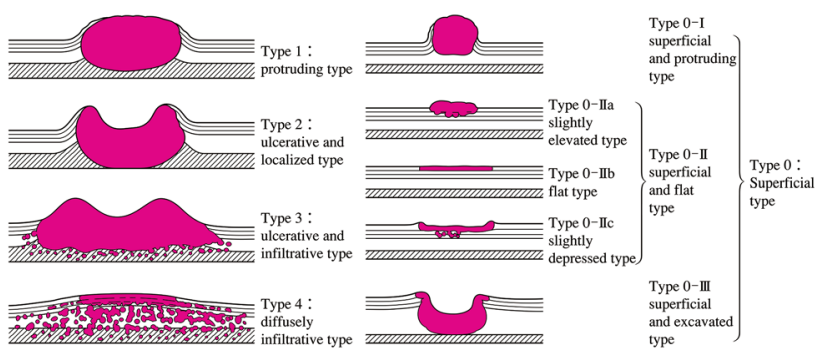

Fig. 1-2 Macroscopic classification (Type 0-4)

determine the order of the depth, the description order depends on the size of the area occupied by the lesion. The largest lesion is described first.

e.g.: MtLt, LtAeG.

\subsubsection{Macroscopic tumor type}

\subsubsection{Principles of tumor type classification}

The tumor type classification is based on the macroscopic findings. Radiological and endoscopic classifications are based on the macroscopic classification.

Tumors in which invasion is macroscopically diagnosed to be limited to within the submucosa are classified as superficial type, while tumors in which invasion is diagnosed to extend to the muscularis propria or beyond are classified as advanced type. The superficial type has the prefix ' 0 ' and is classified into 0 -I, 0 -II or 0 -III. The advanced type is divided into 4 categories: 1 , 2,3 , or 4 . When a tumor cannot be classified into any of the $5(0-4)$ categories or their combinations, it is classified as 5 .

\subsubsection{Macroscopic classification (Fig. 1-2) Note 1}

Type $0 \quad$ Superficial type

Type 1 Protruding type

Type 2 Ulcerative and localized type

Type 3 Ulcerative and infiltrative type

Type 4 Diffusely infiltrative type

Type 5 Unclassifiable type

Type 5a Unclassifiable type without treatment

Type 5b Unclassifiable type after treatment ${ }^{\text {Notes } 1,2}$

Note 1: The macroscopic tumor type before chemotherapy and/or radiotherapy is described. Previous treatment is indicated. Cases with minor changes following treatment and which fit the macroscopic tumor type(s) are classified as type 1-4 and cases of major changes are designated as unclassifiable type.

Note 2: Any former treatment(s) is mentioned before the macroscopic tumor type. e.g.: CT-3, CRT-5b, EMR-0-IIc 


\subsubsection{Subclassification of superficial type (type 0)}

Type 0-I Superficial and protruding type

Type 0-Ip Pedunculated type

Type 0-Is Sessile (broad based) type

Type 0-II Superficial and flat type

Type 0-IIa Slightly elevated type

Type 0-IIb Flat type

Type 0-IIc Slightly depressed type

Type 0-III Superficial and excavated type

Other notations

Note 1: Combined type: When multiple macroscopic tumor types are mixed in one lesion, it is called a combined type. The wider type is described first and types are connected with + . Double quotation marks ("”) are placed around the macroscopic tumor type that has the deepest tumor invasion. In this case, the main macroscopic tumor type is the deepest one. However, when an advanced type is mixed with a superficial type, the advanced type is described first and double quotation marks are unnecessary.

e.g.: 0-IIc+ "0-Is", 3+0-IIc.

Note 2: Superficial spreading type: superficial type $0-\mathrm{II}$ in which the maximal length of the tumor extends $5 \mathrm{~cm}$ or more longitudinally. It may be noted additionally in the macroscopic tumor type.

[Reference]

Japanese Society for Esophageal Diseases. Guidelines for the Clinical and Pathologic Studies on Carcinoma of the Esophagus (in Japanese). 8th ed. Kanehara Shuppan, Tokyo, 1992; 34.

\subsubsection{Depth of tumor invasion $(T)$}

TX Depth of tumor invasion cannot be assessed

T0 No evidence of primary tumor

T1a Tumor invades mucosa ${ }^{\text {Note }}{ }^{1}$ (Fig 1-3)

T1a-EP Carcinoma in situ (Tis)

T1a-LPM Tumor invades lamina propria mucosae (LPM)

T1a-MM Tumor invades muscularis mucosae (MM)

T1b Tumor invades submucosa (SM) ${ }^{\text {Notes 2, 3, } 4}$

T1b-SM1 Tumor invades the upper third of the submucosal layer

T1b-SM2 Tumor invades the middle third of the submucosal layer

T1b-SM3 Tumor invades the lower third of the submucosal layer

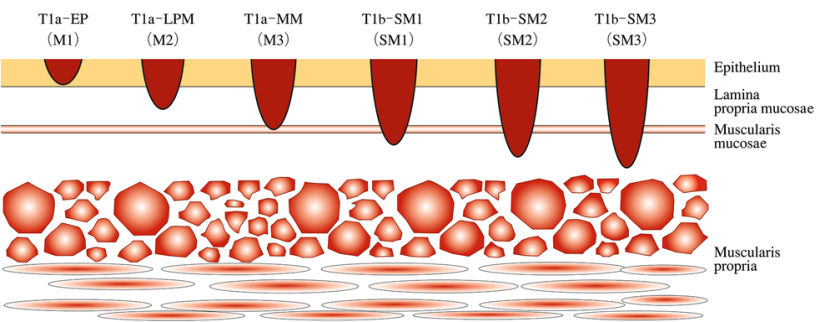

Fig. 1-3 Subclassification for superficial cancer (modified from the guidelines for esophageal cancer treatment)

T2 Tumor invades muscularis propria (MP)

T3 Tumor invades adventitia (AD)

T4 Tumor invades adjacent structures (AI) ${ }^{\text {Notes 5, } 7}$

T4a Pleura, pericardium, diaphragm, lung, thoracic duct, azygos vein, nerve.

T4b Aorta (great artery), trachea, bronchus, pulmonary vein, pulmonary artery, vertebral body.

Note 1: Early esophageal cancer: T1a can be designated as early cancer of the esophagus regardless of the presence or absence of lymph node or distant organ metastasis. e.g.: early esophageal cancer: T1aNxMx.

Note 2: Superficial esophageal cancer: $\mathrm{T} 1 \mathrm{a}$ and $\mathrm{T} 1 \mathrm{~b}$ are designated as superficial cancer regardless of lymph node or distant organ metastasis.

e.g.: superficial esophageal cancer: T1NxMx

Note 3: Formerly used subclassification of superficial type generally corresponds to the following.

M1: T1a-EP, M2: T1a-LPM, M3: T1a-MM, SM1: T1bSM1, SM 2: T1b-SM2, SM 3: T1b-SM3

Note 4: In endoscopically resected specimens, a tumor invading the submucosa to a depth of $200 \mu \mathrm{m}$ or less from the lamina muscularis mucosae is classified as T1bSM1, while a tumor extending more than $200 \mu \mathrm{m}$ is classified as T1b-SM2, since the distance of the submucosal layer is unknown.

Note 5: Invaded organs such as the pericardium, aorta, vena cava, trachea, lung, diaphragm, thoracic duct, recurrent laryngeal nerve, azygos vein should be recorded

e.g.: T4a (lung).

Note 6: When a metastatic lymph node additionally invades a surrounding organ other than the esophagus, it should be classified as T4 and recorded as "T4 (metastatic node number-invaded organ)".

e.g.: T4b (No.112aoA-Aorta). 


\subsection{Metastatic lesions from esophageal cancer}

\subsubsection{Lymph node metastasis}

2.2.1.1. Naming and numbers of lymph node stations

The names and numbers of lymph nodes are defined as shown in Table 1-5 and Fig. 1-4. The stations of cervical and thoracic lymph nodes are shown in Figs. 1-5, 1-6 and 1-7. The names and numbers of abdominal lymph node stations are defined in the Japanese Classification of Gastric Carcinoma (Table 1-5).

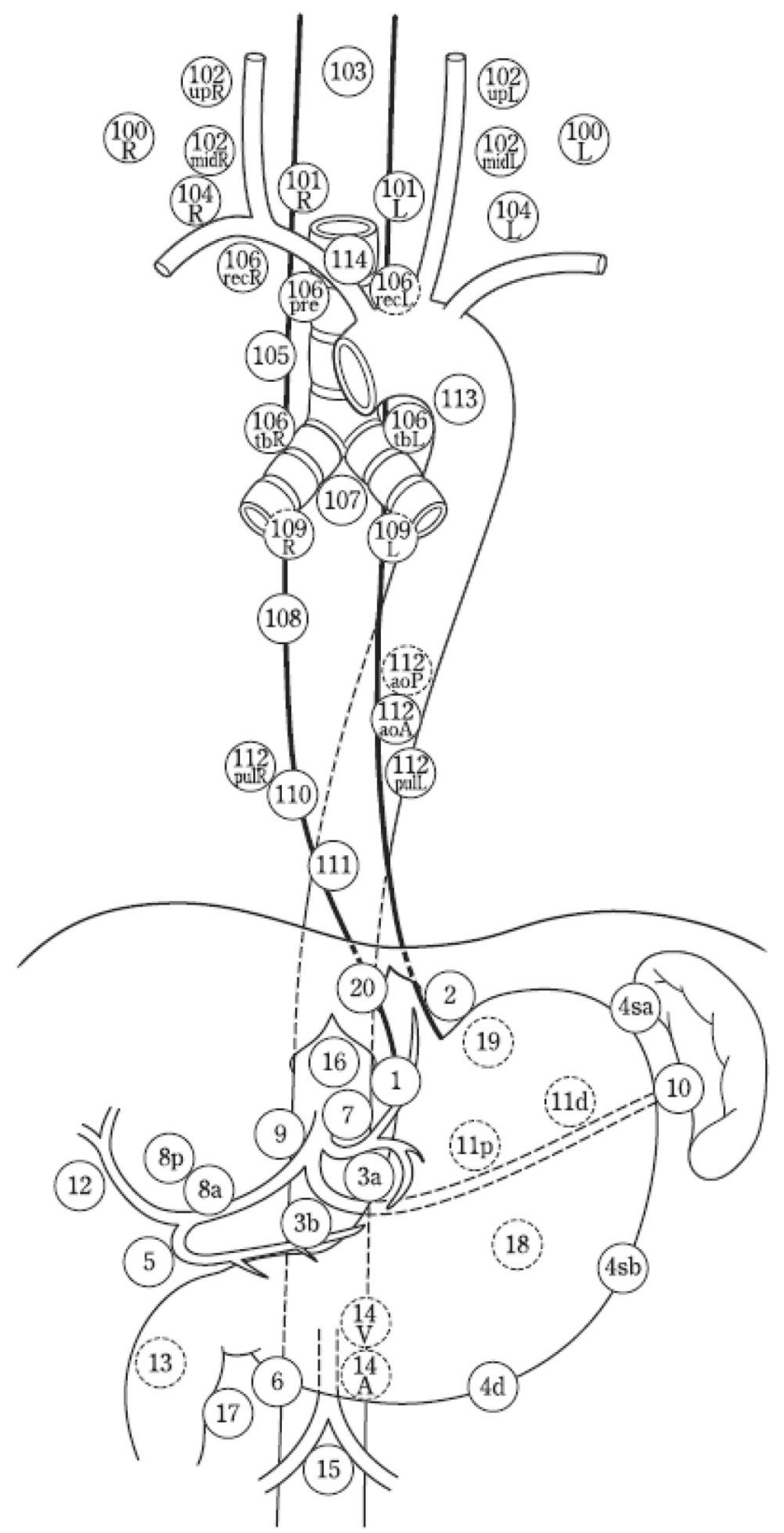

Fig. 1-4 Station numbers of regional lymph nodes
Note: The number of lymph node stations should be recorded using "No." plus a number.

e.g.: No.106recR.

[Reference]

Japanese Gastric Cancer Association. Japanese Classification of Gastric Carcinoma. 14th ed. Kanehara Shuppan, Tokyo, 2010.

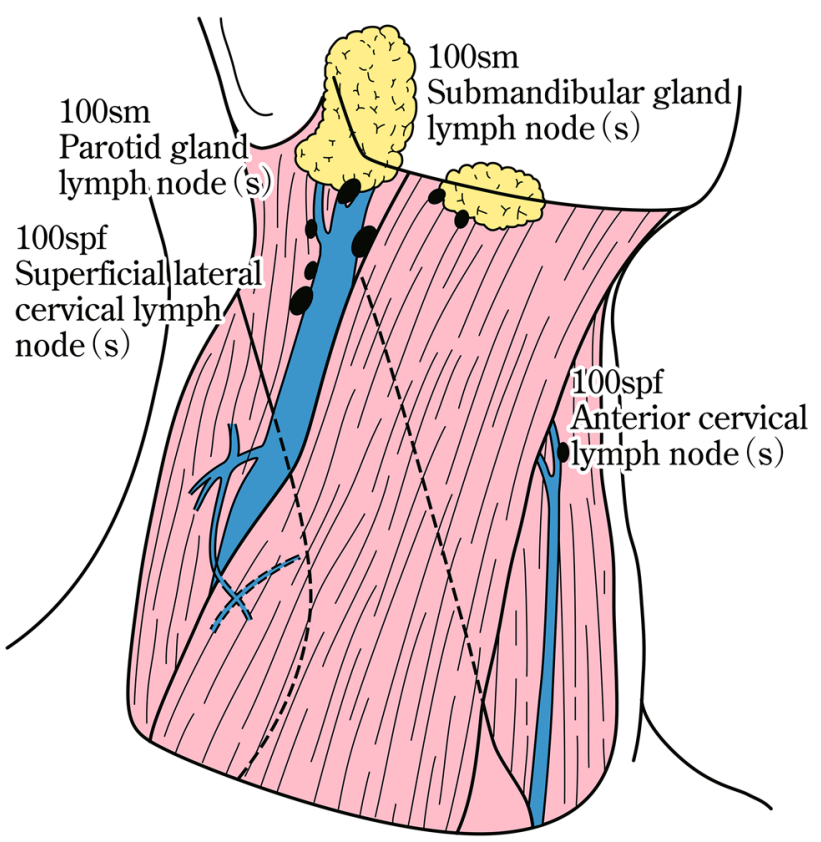

Fig. 1-5 Superficial cervical lymph nodes

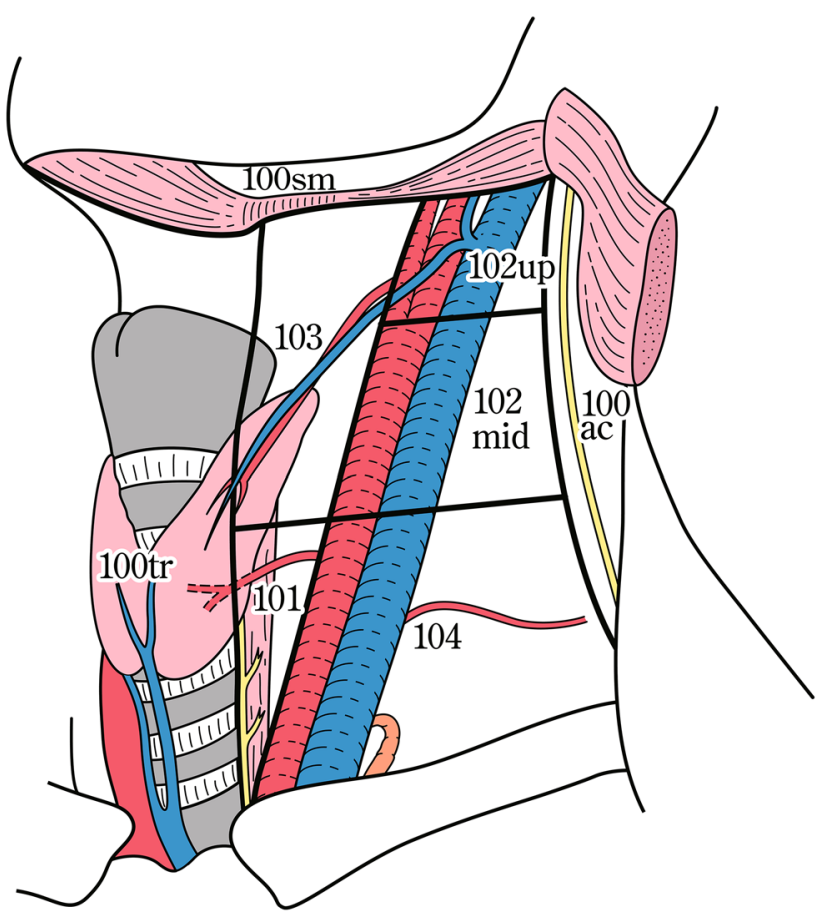

Fig. 1-6 Deep cervical lymph nodes 

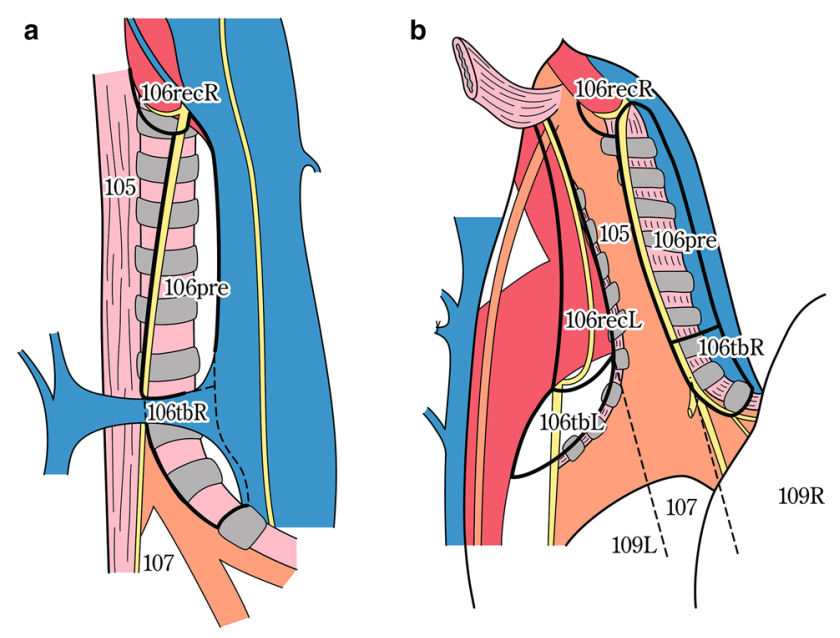

Fig. 1-7 Tracheobronchial lymph nodes (right view of the trachea) (posterior view of the trachea)

Table 1-5 Numbers and naming of regional lymph nodes

\begin{tabular}{ll}
\hline (1) Cervical lymph nodes* (Figs. 1-4, 1-5, 1-6) \\
No. 100 & Superficial lymph nodes of the neck \\
No. 100spf & Superficial cervical lymph nodes \\
No. 100sm & Submandibular lymph nodes \\
No. 100tr & Cervical pretracheal lymph nodes \\
No. 100ac & Accessory nerve lymph nodes \\
No. 101 & Cervical paraesophageal lymph nodes \\
No. 102 & Deep cervical lymph nodes \\
No. 102up & Upper deep cervical lymph nodes \\
No. 102mid & Middle deep cervical lymph nodes \\
No. 103 & Peripharyngeal lymph nodes \\
No. 104 & Supraclavicular lymph nodes \\
(2) Thoracic lymph nodes & \\
(Figs. 1-4, 1-7) & \\
No. 105 & Upper thoracic paraesophageal lymph nodes \\
No. 106 & Thoracic paratracheal lymph nodes \\
No. 106rec & Recurrent nerve lymph nodes \\
No. 106recL & Left recurrent nerve lymph nodes \\
No. 106recR & Right recurrent nerve lymph nodes \\
No. 106pre & Pretracheal lymph nodes \\
No. 106tb & Tracheobronchial lymph nodes \\
No. 106tbL & Left tracheobronchial lymph nodes \\
No. 106tbR & Right tracheobronchial lymph nodes \\
No. 107 & Subcarinal lymph nodes \\
No. 108 & Middle thoracic paraesophageal lymph nodes \\
No. 109 & Main bronchus lymph nodes \\
No. 109L & Left main bronchus lymph nodes \\
No. 109R & Right main bronchus lymph nodes \\
No. 110 & Lower thoracic paraesophageal lymph nodes \\
No. 111 & \\
No. 112 & Supradiaphragmatic lymph nodes \\
No. 112aoA & Po. 112aoP
\end{tabular}

\begin{tabular}{|c|c|}
\hline No. 112pul & Pulmonary ligament lymph nodes \\
\hline No. 113 & $\begin{array}{l}\text { Ligamentum arteriosum lymph nodes (Botallo } \\
\text { lymph nodes) }\end{array}$ \\
\hline No. 114 & Anterior mediastinal lymph nodes \\
\hline \multicolumn{2}{|c|}{ (3) Abdominal lymph nodes (Fig. 1-4) } \\
\hline No. 1 & Right paracardial lymph nodes \\
\hline No. 2 & Left paracardial lymph nodes \\
\hline No. $3 \mathrm{a}$ & $\begin{array}{l}\text { Lesser curvature Lymph nodes along the branches } \\
\text { of the left gastric artery }\end{array}$ \\
\hline No. $3 \mathrm{~b}$ & $\begin{array}{l}\text { Lesser curvature Lymph nodes along the } 2 \text { nd } \\
\text { branches and distal part of the right gastric artery }\end{array}$ \\
\hline No. 4 & Lymph nodes along the greater curvature \\
\hline No. 4 sa & Lymph nodes along the short gastric vessels \\
\hline No. $4 \mathrm{sb}$ & Lymph nodes along the left gastroepiploic artery \\
\hline No. $4 \mathrm{~d}$ & Lymph nodes along the right gastroepiploic artery \\
\hline No. 5 & Suprapyloric lymph nodes \\
\hline No. 6 & Infrapyloric lymph nodes \\
\hline No. 7 & Lymph nodes along the left gastric artery \\
\hline No. $8 \mathrm{a}$ & $\begin{array}{l}\text { Lymph nodes along the common hepatic artery } \\
\text { (anterosuperior group) }\end{array}$ \\
\hline No. $8 \mathrm{p}$ & $\begin{array}{l}\text { Lymph nodes along the common hepatic artery } \\
\text { (Posterior group) }\end{array}$ \\
\hline No. 9 & Lymph nodes along the celiac artery \\
\hline No. 10 & Lymph nodes at the splenic hilum \\
\hline No. 11 & Lymph nodes along the splenic artery \\
\hline No. $11 \mathrm{p}$ & Lymph nodes along the proximal splenic artery \\
\hline No. 11d & Lymph nodes along the distal splenic artery \\
\hline No. 12 & Lymph nodes in the hepatoduodenal ligament \\
\hline No. 13 & $\begin{array}{l}\text { Lymph nodes on the posterior surface of the } \\
\text { pancreatic head }\end{array}$ \\
\hline No. 14 & Lymph nodes along the superior mesenteric vessels \\
\hline No. $14 \mathrm{~A}$ & Lymph nodes along the superior mesenteric artery \\
\hline No. $14 \mathrm{~V}$ & Lymph nodes along the superior mesenteric vein \\
\hline No. 15 & Lymph nodes along the middle colic artery \\
\hline No. 16 & Lymph nodes around the abdominal aorta \\
\hline No. $16 \mathrm{a} 1$ & Lymph nodes in the aortic hiatus \\
\hline No. $16 \mathrm{a} 2$ & $\begin{array}{l}\text { Lymph nodes around the abdominal aorta (from the } \\
\text { upper margin of the celiac trunk to the lower } \\
\text { margin of the left renal vein) }\end{array}$ \\
\hline No. $16 \mathrm{~b} 1$ & $\begin{array}{l}\text { Lymph nodes around the abdominal aorta (from the } \\
\text { lower margin of the left renal vein to the upper } \\
\text { margin of the inferior mesenteric artery) }\end{array}$ \\
\hline No. $16 \mathrm{~b} 2$ & $\begin{array}{l}\text { Lymph nodes around the abdominal aorta (from the } \\
\text { upper margin of the inferior mesenteric artery to } \\
\text { the aortic bifurcation) }\end{array}$ \\
\hline No. 17 & $\begin{array}{l}\text { Lymph nodes on the anterior surface of the } \\
\text { pancreatic head }\end{array}$ \\
\hline No. 18 & $\begin{array}{l}\text { Lymph nodes along the inferior margin of the } \\
\text { pancreas }\end{array}$ \\
\hline No. 19 & Infradiaphragmatic lymph nodes \\
\hline No. 20 & $\begin{array}{l}\text { Lymph nodes in the esophageal hiatus of the } \\
\text { diaphragm }\end{array}$ \\
\hline
\end{tabular}

The left side (L) and the right side (R) should be distinguished for 101, 102, 104, 106rec, 106tb, 109, and 112pul 
Table 1-6 Lymph node groups according to the location of the tumor

\begin{tabular}{|c|c|c|c|}
\hline Tumor location & Group 1 (N1) & Group 2 (N2) & Group 3 (N3) \\
\hline Cervical Ce & $101,106 \mathrm{rec}^{\mathrm{a}}$ & $102,104,105^{\mathrm{a}}$ & 100 \\
\hline Upper thoracic Ut & $101,105,106 \mathrm{rec}$ & 104, 106tbL, 107, 108, 109 & $\begin{array}{l}\text { 102mid, 106pre, 106tbR, 110, 112aoA, 112pul, } \\
1,2,3 \mathrm{a}, 7,20\end{array}$ \\
\hline Middle thoracic Mt & 106rec, $108,1,2,3 \mathrm{a}$ & $\begin{array}{l}\text { 101, 104, 105, 107, 109, 110, 112aoA, } \\
\text { 112pul, 7, 9, } 20\end{array}$ & $106 \mathrm{tbL}$ \\
\hline Lower thoracic Lt & $110,1,2,3 a, 7,20$ & $\begin{array}{l}\text { 101, 106rec, 107, 108, 109, 112aoA, 112pul, } \\
\quad 9\end{array}$ & $104,105,106 \mathrm{tbL}, 111,8 \mathrm{a}, 11 \mathrm{p}$ \\
\hline Abdominal Ae & $110,1,2,3 a, 7,20$ & 111, 112aoA, 112pul, 8a, 9, 11p, 19 & 106rec, 107, 108, 109, 11d \\
\hline
\end{tabular}

Nodes other than N1 through N3 are expressed as N4

${ }^{a}$ Limited to the area which can be dissected from the cervical incision

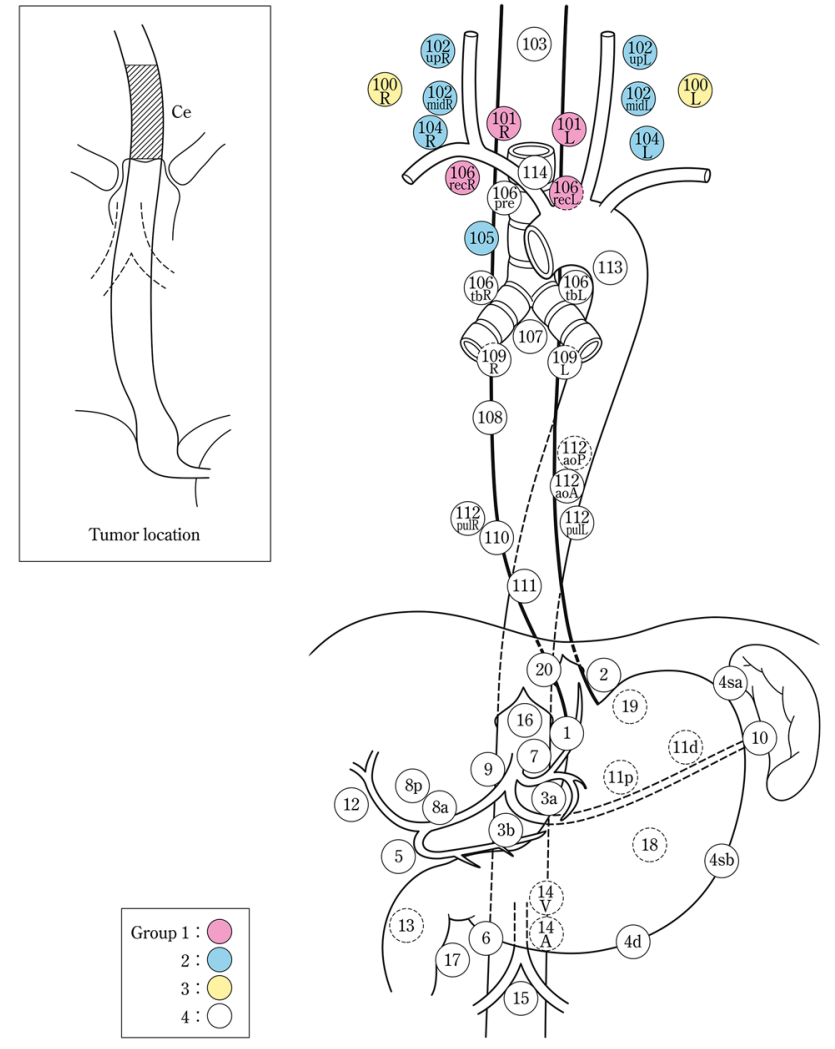

Fig. 1-8 Lymph node groups for tumors located in $\mathrm{Ce}$

\subsubsection{Lymph node groups}

Lymph node groups are defined according to the location of the tumor as shown in Table 1-6, Figs. 1-8, 1-9, 1-10, 111 and 1-12.

Note: In deciding the lymph node group of multiple esophageal cancers and widely extending esophageal cancer, the location of the deepest tumor invasion takes precedence in documentation.
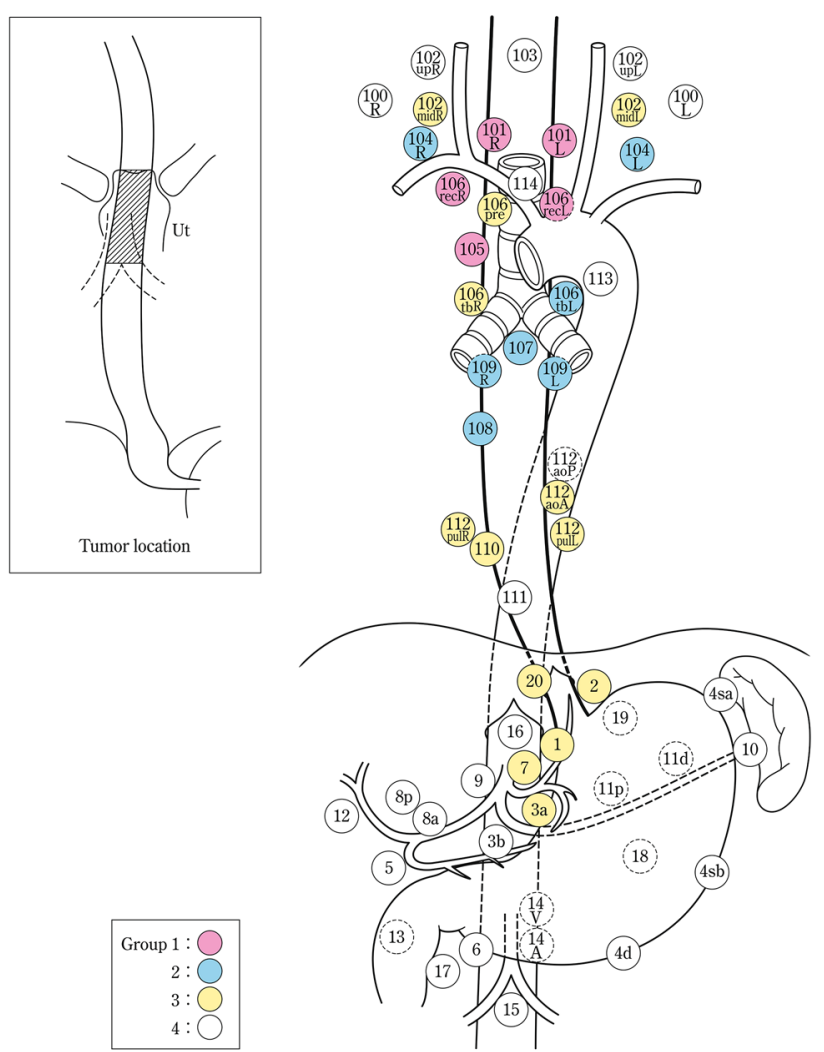

Fig. 1-9 Lymph node groups of tumors located in Ut

\subsubsection{Grading of lymph node metastasis $(\mathrm{N})$}

NX Lymph node metastasis cannot be assessed

N0 No lymph node metastasis

N1 Metastasis involving only Group 1 lymph nodes

N2 Metastasis to Group 2 lymph nodes, regardless of involvement of Group 1 lymph nodes

N3 Metastasis to Group 3 lymph nodes, regardless of involvement of Group 1 or 2 lymph nodes

N4 Metastasis to distant (Group 4) lymph nodes, regardless of whether any other group(s) of regional lymph nodes are involved or not 


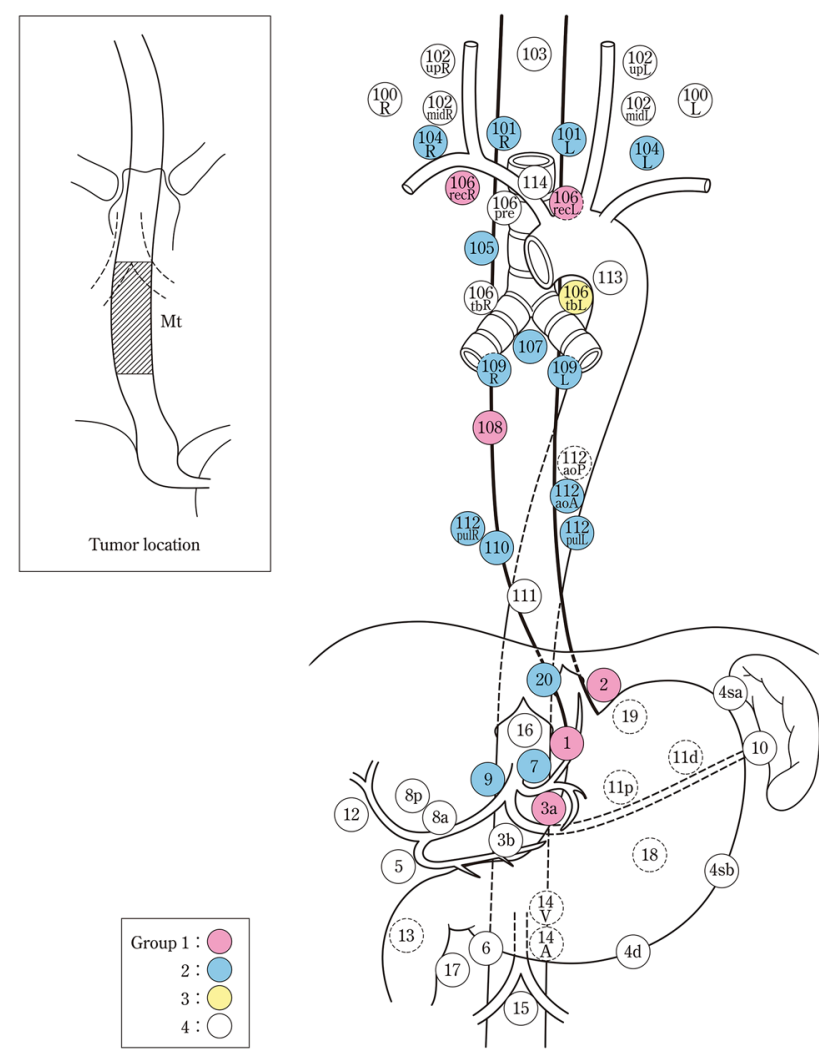

Fig. 1-10 Lymph node groups for tumors located in Mt
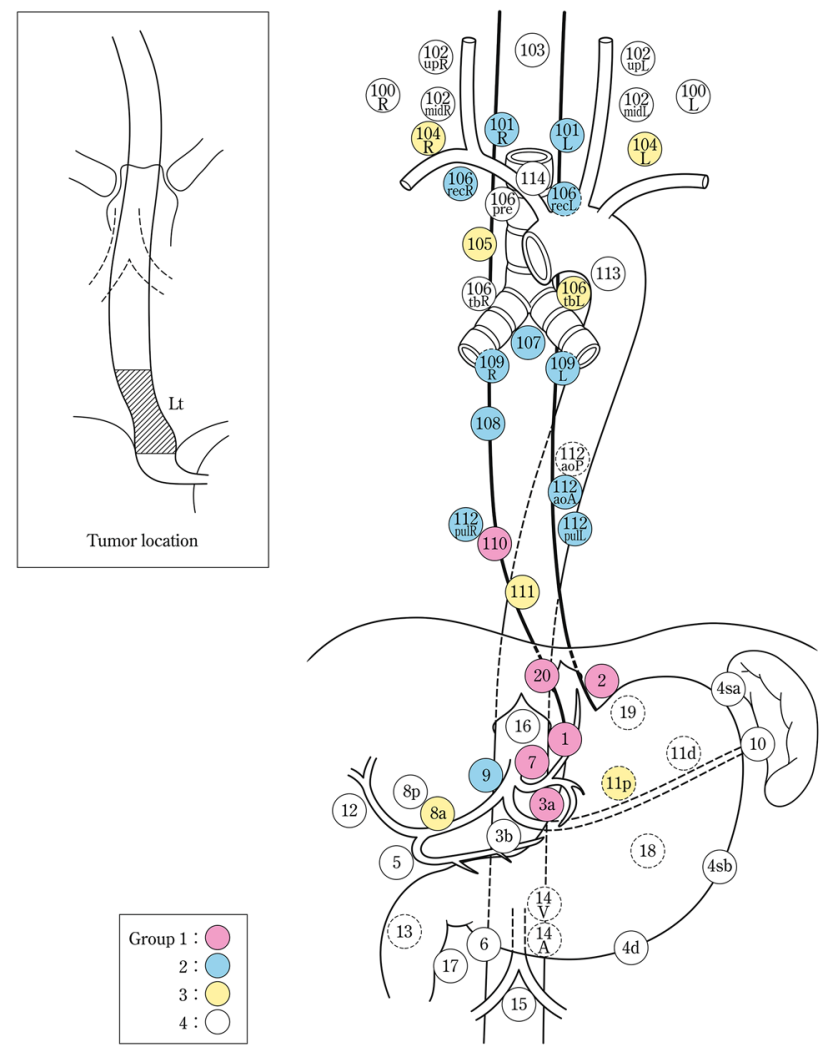

Fig. 1-11 Lymph node groups for tumors located in Lt
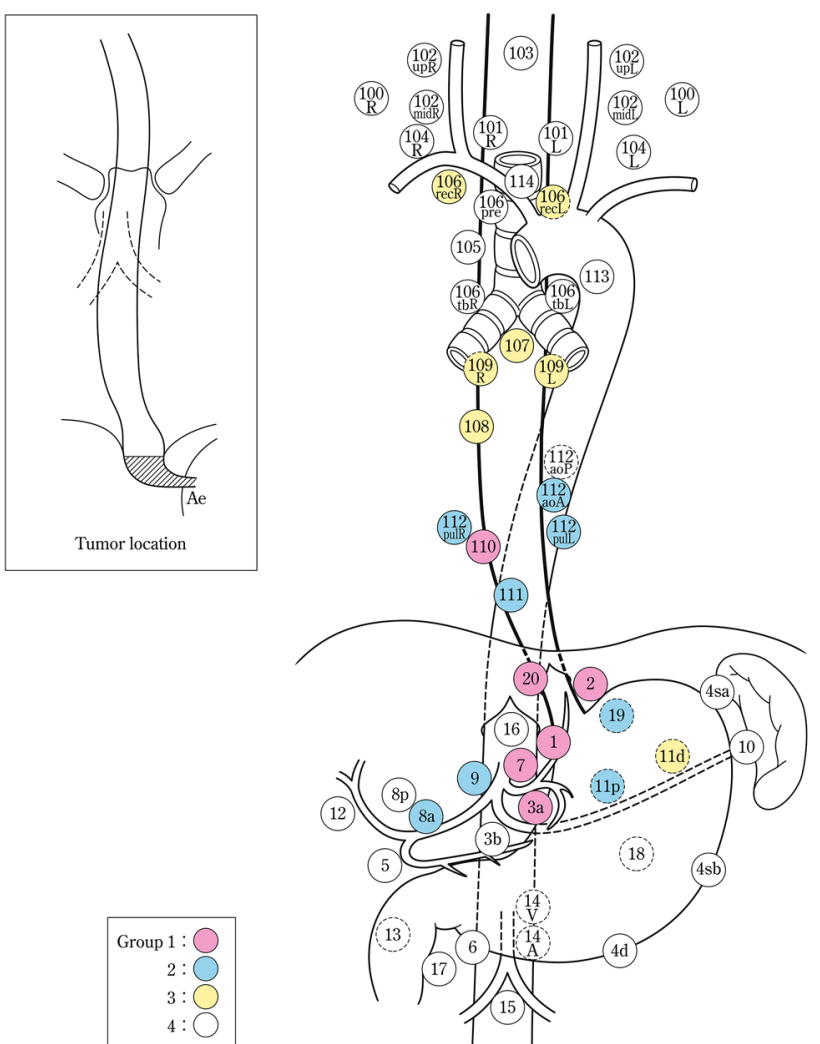

Fig. 1-12 Lymph node groups for tumors located in Ae (EG)

Note: Extralymph node metastasis (tumor nodule) is included within $\mathrm{N}$.

\subsubsection{Distant organ metastasis (M)}

MX Distant organ metastasis cannot be assessed

M0 No distant organ metastasis

M1 Distant organ metastasis

Note 1: Organs with metastasis should be recorded in parentheses.

e.g.: M1 (lung), M1 (liver, stomach).

Note 2: Pleural, peritoneal, and pericardial dissemination should be recorded as M1.

\subsection{Stage (Table 1-7)}

The stage should be recorded based on the following TNM stage classification.

e.g.: T2N2M0, Stage III.

\subsection{Multiple primary cancers}

Multiple primary cancers of the esophagus:

The term "multiple primary cancers of the esophagus" is used to refer to the presence of two or more primary esophageal cancers. 
Table 1-7 Stage

\begin{tabular}{|c|c|c|c|c|c|c|}
\hline $\begin{array}{l}\text { Depth } \\
\text { of tumor invasion }\end{array}$ & N0 & N1 & N2 & N3 & N4 & M1 \\
\hline T0, T1a & 0 & II & II & III & IVa & $\mathrm{IVb}$ \\
\hline $\mathrm{T} 1 \mathrm{~b}$ & I & II & II & III & IVa & $\mathrm{IVb}$ \\
\hline $\mathrm{T} 2$ & II & II & III & III & IVa & $\mathrm{IVb}$ \\
\hline $\mathrm{T} 3$ & II & III & III & III & IVa & $\mathrm{IVb}$ \\
\hline $\mathrm{T} 4 \mathrm{a}$ & III & III & III & III & IVa & $\mathrm{IVb}$ \\
\hline $\mathrm{T} 4 \mathrm{~b}$ & IVa & IVa & IVa & IVa & IVa & $\mathrm{IVb}$ \\
\hline
\end{tabular}

T4a pleura, pericardium, diaphragm, lung, thoracic duct, azygos vein, nerve

$T 4 b$ aorta (large vessel), trachea, bronchus, pulmonary vein, pulmonary artery, vertebra

Note: Descriptions of the locations of multiple primary cancers of the esophagus should be made according to the order of the depth of tumor invasion (deeper to shallower), inserting "/" between the abbreviations for the location of each lesion; the total number of lesions should also be recorded in parentheses.

e.g.: $\mathrm{MtUt} / \mathrm{Lt} / \mathrm{Lt}$ (3 lesions).

Multi-organ primary cancers including the esophagus:

The term "multi-organ primary cancers including the esophagus" is used to refer to the presence of one or more primary malignant diseases other than esophageal cancer in a patient with primary esophageal cancer.

Multiple primary cancers including the esophagus:

The term "multiple primary cancers including the esophagus" indicates the concept combining both "multiple primary cancers of the esophagus" and "multi-organ primary cancers including the esophagus".

Note 1: In cases with multi-organ primary cancers including the esophagus, organs other than the esophagus should be specified in parentheses.

Note 2: Whether the multiplicity is synchronous or metachronous should be recorded.

e.g.: Multi-organ primary cancers: stomach (synchronous).

\section{Surgical aspects}

\subsection{Handling of the resected specimen}

The resected esophagus should be cut and opened along the longitudinal line on the side opposite to the lesion. The opened esophagus should be gently stretched longitudinally and fixed so that the length of the specimen becomes similar to its size in vivo. The specimen should be treated with iodine solution after fixation in order to accurately describe the macroscopic findings. This is particularly important in superficial carcinoma. Photographic recording is recommended for both fresh and fixed specimens.

3.2. Description of surgical findings and macroscopic findings of primary tumor

Operative findings should be identified in the record putting "s" in front of each factor.

e.g.: sT2, sStageII.

\subsubsection{Tumor size (Fig. 1-13)}

The greatest longitudinal dimension in millimeters and the greatest transverse (at $90^{\circ}$ to the longitudinal tumor axis) dimension in millimeters: $\mathrm{a} \times \mathrm{b}(\mathrm{mm})$

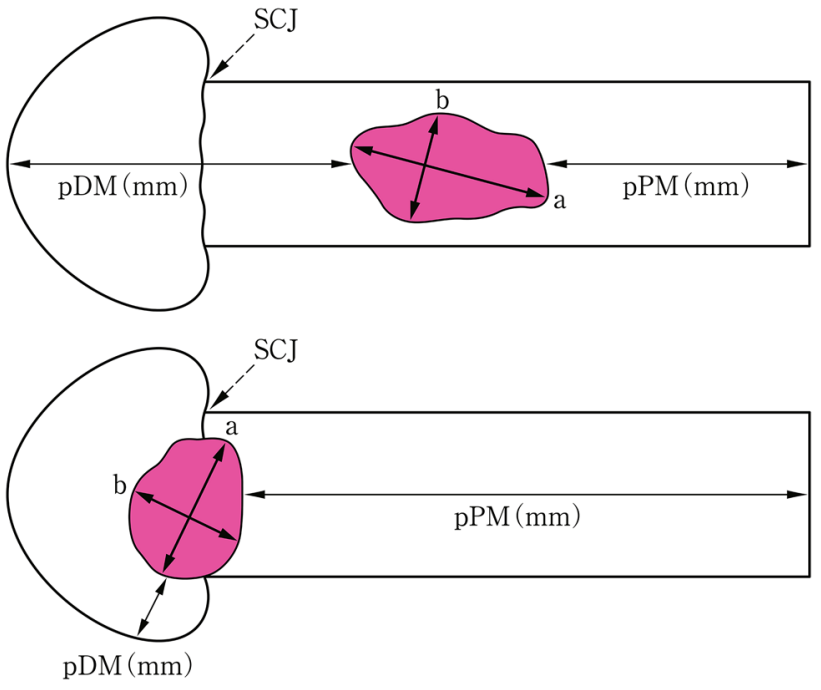

Fig. 13 Tumor size and the distance from resection margin to tumor. $a$ Greatest longitudinal dimension $(\mathrm{mm}) . \quad b$ Greatest transverse dimension $(\mathrm{mm})$ 


\subsubsection{Distance from surgical margin to the tumor}

(Fig. 1-13)

Proximal (oral) margin (PM) (mm)

Distal (anal) margin (DM) (mm)

\subsubsection{Macroscopic tumor type}

The macroscopic appearance of tumors before and after fixation can be different. Under such circumstances, the macroscopic tumor type should be described according to prefixation observations, and the pathological tumor type should be described based on the post-fixation findings. Pathological tumor types can be classified referring to the cross-sectional observation. Macroscopic tumor types should be determined regardless of microscopic depth of tumor invasion.

Note: The presence of preoperative chemotherapy and radiotherapy should be recorded with the macroscopic tumor type.

\subsubsection{Surgical margin}

\subsubsection{PM: Proximal (oral) margin}

PMX Proximal margin cannot be assessed

PM0 No evidence of tumor invasion

PM1 Tumor invasion

\subsubsection{DM: Distal (anal) margin}

DMX Distal margin cannot be assessed

DM0 No evidence of tumor invasion

DM1 Tumor invasion

Note: The distance from the resection margin to tumor should be recorded in millimeters for PM0 and DM0 specimens.

\subsubsection{RM: Radial margin}

RMX Radial margin cannot be assessed

RM0 No evidence of tumor invasion

RM1 Tumor invasion

Note: The radial margin is the surgical margin in the radial direction, i.e., the outer surface of the surgical dissection plane.

3.3. Intramural metastasis and multiple cancers in the esophagus

\subsubsection{IM: Intramural metastasis}

Metastatic lesions in the esophageal, pharyngeal, or gastric wall macroscopically (clearly) separate from the primary tumor should be recorded as IM, and the number of such lesions should be described.

IMX Intramural metastasis cannot be assessed

IM0 No intramural metastasis

IM1 Intramural metastasis

Note: IM in the gastric wall should be recorded as "IM1St". It is classified as organ metastasis (M1).

\subsubsection{Multiple cancers of the esophagus}

Multiple cancers are two or more primary cancer lesions separate from each other. Multiple cancers and IM should be clearly differentiated in the description.

\subsection{Lymph nodes}

\subsubsection{Preparation of resected lymph nodes for pathologi-} cal examination

Surgically dissected lymph nodes are classified according to the definition of regional lymph nodes, given individual names or numbers and sent to pathologists. The lymph nodes dissected en bloc with the esophagus should be isolated from the specimen before fixation.

\subsubsection{Grading of lymph node metastasis $(N)$}

The surgical diagnosis of the grading of lymph node metastasis $(\mathrm{sN})$ should be made comprehensively with intraoperative findings of macroscopic observation, imaging examinations, immediate pathological diagnosis with frozen section, and macroscopic findings obtained during postoperative preparation.

\subsubsection{Lymph node dissection (D)}

\subsubsection{Field of lymph node dissection}

Three-field dissection

Two-field dissection
Dissection of cervical, thoracic and abdominal lymph nodes through cervical, thoracic and abdominal approaches, respectively ${ }^{\text {Note }}$ Dissection of thoracic and abdominal lymph nodes through thoracic and abdominal approaches, respectively.Dissection of cervical and abdominal lymph nodes through cervical and abdominal approaches, respectively.Dissection of cervical and thoracic lymph nodes through cervical and thoracic approaches, respectively. 
One-field

dissection
Dissection of a single field of cervical, thoracic and abdominal lymph nodes through cervical, thoracic or abdominal approaches, respectively.

Note: The term "three-field dissection" should not be applied when only the cervical paraesophageal nodes $(101 \mathrm{R}, 101 \mathrm{~L})$ are dissected in the neck.

\subsubsection{Extent of lymph node dissection (D)}

DX Extent of lymph node dissection cannot be assessed.

D0 No or incomplete dissection of Group-1 lymph nodes.

D1 Complete dissection of Group-1 lymph nodes, but no or incomplete dissection of Group-2 lymph nodes.

D2 Complete dissection of Group-1 and Group-2 lymph nodes, but no or incomplete dissection of Group-3 lymph nodes.

D3 Complete dissection of Group-1, Group-2 and Group-3 lymph nodes

\subsection{Distant organ metastasis (M)}

Surgical findings of distant organ metastasis (sM) should be determined through comprehensive consideration of operative macroscopic findings, intraoperative imaging examinations such as intraoperative ultrasound examination, macroscopic observation of resected specimen, and intraoperative immediate pathological diagnosis with frozen section. Whether the distant organ metastasis was resected or not should be recorded.

3.6. Residual tumor (R) ${ }^{\text {Note } 1}$ (Fig. 1-14)

RX Presence of residual tumor cannot be assessed.

R0 No residual tumor.

R1 Microscopic residual tumor ${ }^{\text {Note } 2}$

R2 Macroscopic residual tumor ${ }^{\text {Note } 3}$

Note 1: The postoperative state of both primary tumor and metastatic lesions should be evaluated.

Note 2: This refers to the presence of a tumor on the surgical margin of the resected specimen that was identified upon microscopic examination.

Note 3: This refers to a macroscopically obvious residual tumor.

\subsection{Curativity (Cur) (Table 1-8)}

Cur A Complete removal of the tumor is strongly believed.

sStage 0-III, and sR0, and sD > sN (Fig. 1-14).
Table 1-8 Surgical curativity

\begin{tabular}{llclc}
\hline & Stage & N and D & PM, DM, RM & R \\
\hline Cur A & $\begin{array}{l}\text { Stage 0, I, II, III } \\
\text { Cur B }>\text { N }\end{array}$ & PM0, DM0, RM0 & R0 \\
Cur C & $\begin{array}{l}\text { Residher Cur A nor Cur C } \\
\text { R2 }\end{array}$ & & \\
& Pal tumor assessed by surgical (macroscopic) findings, \\
\hline
\end{tabular}

Cur B Neither Cur A nor Cur C R1. sStage IVa, sStage IVb or $\mathrm{sD} \leqq \mathrm{sN}$, but $\mathrm{R} 0$ was achieved with resection of a $\mathrm{T} 4 \mathrm{~b}$ tumor or complete removal of metastatic tumor (M1) or lymph nodes.

Cur C Residual tumor.

R2, i.e., M1 evident residual tumor in distant organ(s) (M1), lymph nodes, or surgical margin(s) (PM1, DM1, RM1).

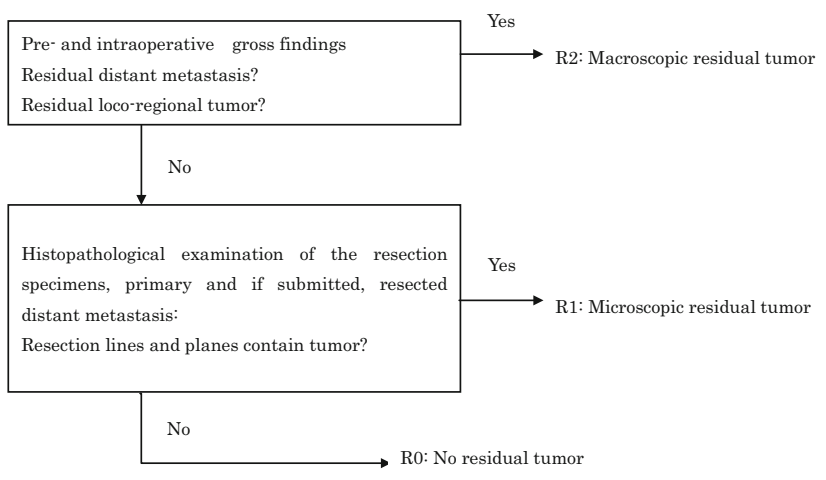

Fig. 1-14 Curativity

\section{Pathological findings}

4.1. Handling of the surgically resected specimens (Fig. 1-15)

Before cutting the resected esophagus, the formalin-fixed specimen should be treated with iodine solution to confirm the unstained area. Rinsing the sample with tap water for at least $1 \mathrm{~h}$ can result in a good staining condition. To increase the contrast between stained and unstained areas, the sample should be treated with a relatively low concentration $(0.1-0.5 \%)$ of iodine solution for a long time.

The resected specimen should be cut parallel along the long axis of the esophagus. Whole step sections are made in superficial type cancer. One representative section of an advanced tumor at the site of deepest invasion, parallel or perpendicular to the esophagus should be blocked and used for microscopic examination. Schemas or photographs of the sites of cut sections should be preserved. 

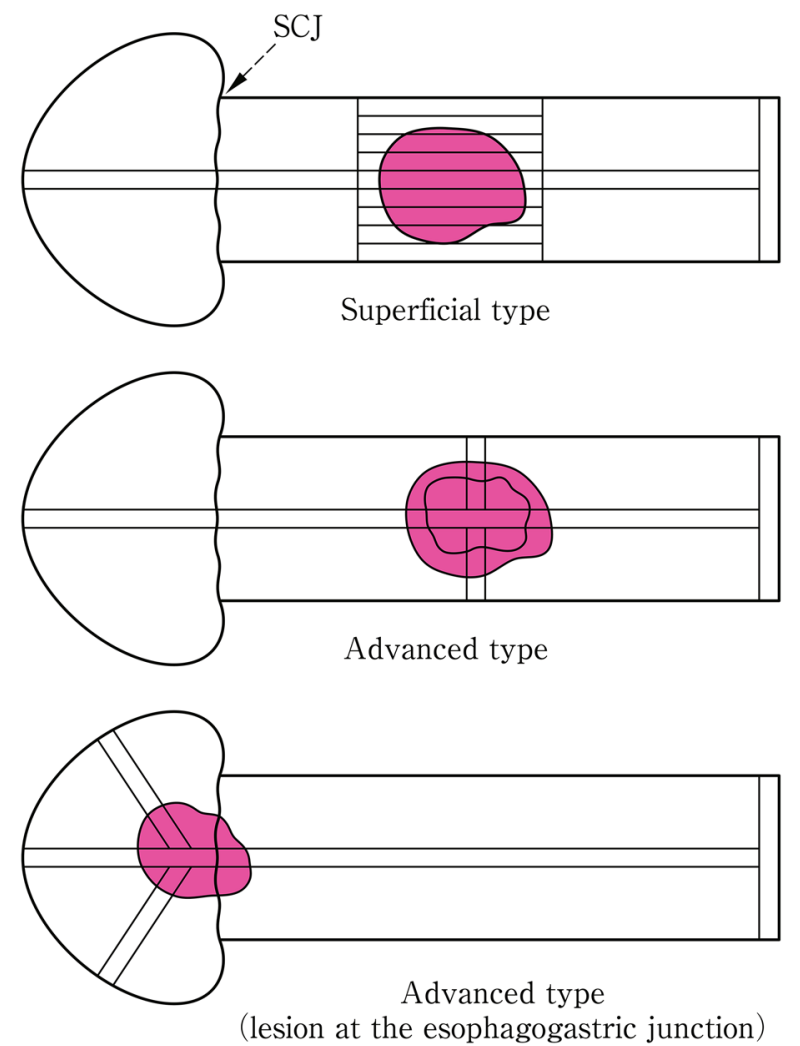

Fig. 1-15 How to cut surgically resected specimens

\subsection{Description of pathological findings}

The $\mathrm{p}$ (pathology) mark is prefixed to the pathological findings except for vascular invasion as follows.

e.g.: p0-Is, pType 2, pT2, pStagedII.

\subsubsection{Histological classification}

4.2.1.1. Benign epithelial neoplasms ${ }^{\text {Note } 1}$

1. Squamous cell papilloma

2. Adenoma

3. Others

4.2.1.2. Intraepithelial neoplasias ${ }^{\text {Note } 2-7}$

1. Squamous intraepithelial neoplasia

\subsubsection{Malignant epithelial neoplasms}

1. Squamous cell carcinoma
a. Well differentiated
b. Moderately differentiated
c. Poorly differentiated

2. Basaloid (-squamous) carcinoma

3. Carcinosarcoma

4. Adenocarcinoma
a. Well differentiated
b. Moderately differentiated
c. Poorly differentiated

5. Adenosquamous carcinoma

6. Mucoepidermoid carcinoma

7. Adenoid cystic carcinoma

8. Neuroendocrine cell tumor ${ }^{\text {Note } 8}$
a. Neuroendocrine tumor (NET) G1 or G2
b. Neuroendocrine carcinoma

9. Undifferentiated carcinoma

10. Others

\subsubsection{Non-epithelial tumors}

1. Smooth muscle tumor

2. Gastrointestinal stromal tumor (GIST)

3. Neurogenic tumor

Schwannoma, neurofibroma, granular cell tumor.

4. Others

Hemangioma, lymphangioma, lipoma, etc.

4.2.1.5. Lymphoid tumors

The definition is according to the WHO classification.

[Reference]

Swerdlow SH, Campo E, Harris NL, et al. WHO Classification of Tumours of Haematopoietic and Lymphoid Tissues, fourth edition. IARC, Lyon, 2008.

\subsubsection{Other malignant tumors}

1. Malignant melanoma

2. Others

\subsubsection{Tumor-like lesions}

Ectopic gastric mucosa

Heterotopic sebaceous gland

Cowden disease

Glycogenic acanthosis

Fibrovascular polyp

Note 1: Squamous papilloma is not a true neoplasia, but reactive hyperplasia.

Note 2: Adenocarcinoma and a tumor-like lesion arising from Barrett mucosa are excluded. The classification of adenocarcinoma in Barrett esophagus is the same as that in the Japanese Classification of Gastric Carcinoma.

Note 3: According to the WHO classification, high-grade intraepithelial neoplasia cannot be diagnosed as carcinoma because of the absence of invasion. In the 11th edition, however, intraepithelial squamous cell 
carcinoma (pT1a-EP carcinoma) or squamous cell carcinoma in situ can be diagnosed when cellular and structural atypia are sufficient to suggest malignancy. The 10th edition mentioned that low-grade intraepithelial neoplasia might contain basal-type squamous cell carcinoma. When such lesions are distributed in the lower half of the epithelium and are sufficiently atypical to suggest malignancy, the lesion can be diagnosed as squamous cell carcinoma according to the classification of the 11 th edition.

Note 4: Most "squamous intraepithelial neoplasias" according to the definition of the 11th edition are endoscopically or macroscopically recognized as a "small unstained or tan-stained area". The lesion may be solitary or multiple.

Note 5: According to the definition of the 11th edition, intraepithelial neoplastic lesion without atypia sufficient to suggest malignancy is termed as squamous intraepithelial neoplasia. Thus, intraepithelial neoplasia does not include squamous cell carcinoma in situ. Please be careful to note the differences in the definitions of intraepithelial neoplasia between the 10th and 11th editions. A two-tier subclassification of intraepithelial neoplasia (low grade and high grade) is not used in the 11th edition. In making a diagnosis of intraepithelial neoplasia based on a biopsy specimen, the inclusion of one of the following comments is recommended: "follow-up is needed", "re-biopsy after a short time period should be recommended", or "immediate rebiopsy is strongly recommended because of suspicious carcinoma".

Note 6: When the determination of a biopsy specimen as "neoplastic" or "reactive" is difficult, the specimen should not be diagnosed as "intraepithelial neoplasia", but rather as "atypical epithelium" or "atypical epithelium, indefinite for neoplasia". For clinicians, the inclusion of instructions such as the need for a re-biopsy is recommended.

Note 7: Squamous cell carcinoma, which is limited to within the epithelial layer without invasion, is different from squamous intraepithelial neoplasia. Squamous cell carcinoma in situ is equal to squamous cell carcinoma with a depth of pT1a-EP.

Note 8: Neuroendocrine tumor and neuroendocrine carcinoma are formally classified as carcinoid tumor and endocrine cell carcinoma, respectively. These terms have been adopted according to the WHO classification. In Japan, however, "endocrine cell carcinoma" is considered to be the correct term, since endocrine cells in the gastrointestinal tract originate from gastrointestinal stem cells.

\subsubsection{Depth of tumor invasion $(p T)^{\text {Note 1-7 }}$}

Note 1: Intraductal spreading of cancer is categorized as pT1a-EP, and if the tumor invades beyond the duct of the esophageal gland, the depth of tumor invasion is defined as the layer presenting extraductal invasion of cancer.

Note 2: The vertical depth of submucosal invasion is measured from the muscularis mucosae, and the depth is recorded in parentheses.

e.g.: pT1b-SM2 (400 $\mu \mathrm{m})$.

Note 3: The depth of tumor invasion is defined histologically as the point of deepest direct invasion by the primary tumor. Vascular invasion within the confines of the primary tumor should be regarded as the depth of direct tumor invasion. However, when vascular invasion is found outside the confines of the primary tumor, the depth of such invasion should be specified in parentheses after the depth of direct invasion. For example, when a primary tumor has invaded into the submucosa (pT1b) but lymphatic invasion is found in the muscularis propria outside the main tumor, this is designated as pT1b (ly-T2). Note 4: Cancer that has macroscopically invaded adjacent organ(s) (sT4) and histologically diagnosed malignant tissue recognized on the surgical radial margin (pRM1) are categorized as pT4.

Note 5: Direct invasion of tumor from lymph node metastasis to the adjacent organ(s) is categorized as pT4. e.g.: Direct invasion from No.108 lymph node metastasis to the lung: pN1 (108-lung) T4a

Note 6: In determining the depth of invasion of an advanced cancer after preoperative treatment, both the depth of invasion by residual tumor and the estimated depth of tumor invasion prior to treatment should be considered. The type of adjuvant therapy (RT-, CT-, CRT-, EMR-), depth of invasion by the residual tumor, and estimated depth of tumor invasion prior to treatment should be specified in the given order, with the last item in parentheses. e.g.: RT-pT1b (T4).

Note 7: If no residual tumor is found in an entire specimen after preoperative treatment, the designation should be pT0, and its stage is recognized as the same as T1a.

e.g.: CRT-pT0 (T3), N0, M0, CRT-pStage 0.

\subsubsection{Infiltrative growth pattern (INF)}

The growth and infiltrative pattern of tumor can be classified into one of the following three types, with regard to the predominant pattern observed at tumor margins. 
INFa (expansive type)

$\mathrm{INFb}$

(intermediate

type)

INFc

(infiltrative

type)

\subsubsection{Vascular invasion $(l y / v)^{\text {Note } 1}$}

Note 1: Indefinite for determination of lymphatic or venous invasion is described as $1 \mathrm{y} / \mathrm{v}$.

4.2.4.1. Lymphatic invasion (ly $)^{\text {Note } 1}$
ly0 None
ly1 Slight
ly2 Moderate
ly3 Severe Notes $2-3^{-3}$

Note 1: Examination using immunohistochemical staining with an anti-D2-40 antibody should be described. e.g.: ly1 (D2-40).

Note 2: Carcinomatous lymphangiosis in distant organ(s) is categorized as M1.

Note 3: A tumor mass found in the thoracic duct is described as positive lymphatic invasion.

4.2.4.2. Venous invasion (v) ${ }^{\text {Note } 1}$

v0 None

v1 Slight

v2 Moderate

v3 Severe

Note 1: Examination by elastic fiber staining methods should be described.

e.g.: v1 (Elastica van Gieson), v2 (Victoria blue).

\subsubsection{Intramural metastasis (pIM)}

\subsubsection{Distance from Surgical margin}

4.2.6.1. Proximal and distal margin (pPM, pDM) ${ }^{\text {Note }}$

Note: The distance from surgical margin to tumor edge in $\mathrm{PPM} 0$ or $\mathrm{pDM} 0$ is measured in histological specimens $(\mathrm{mm})$.
4.2.6.2. Radial margin (pRM)

\subsubsection{Multiple primary cancers}

Present (number of lesions).

Absent.

Note: A lesion with a histological type different from that of the main tumor or an isolated lesion with an intraepithelial component is recognized as another primary cancer, and the patient is classified as having multiple primary cancers.

\subsubsection{Others}

1. Metastatic or invasive cancer from other organs.

2. Co-existing tumor. Leiomyoma, etc.

3. Other non-neoplastic lesions.

Barrett esophagus, Achalasia, etc.

4.2.9. Pathological criteria for the effects of radiation and/ or chemotherapy (Fig. 1-16)

In cases of preoperative radiation and/or chemotherapy, the radiation dose and method of administration, type and dose of chemotherapy, and time interval between preoperative therapy and surgical resection of the tumor are described. In cases of preoperative treatment, all the specimens in which the primary tumor is macroscopically possibly present should be examined histologically.

Grade 0: ineffective

No recognizable cytological or histological therapeutic effect.

Grade 1: slightly effective

Apparently viable cancer cells (including cells having eosinophilic cytoplasm with vacuolation and swollen nuclei) account for $1 / 3$ or more of tumor tissue, but there is some evidence of degeneration of cancer tissue or cells.

Grade 1a: Viable cancer cells accounting for $2 / 3$ or more tumor tissue.

Grade 1b: Viable cancer cells accounting for $1 / 3$ or more, but less than $2 / 3$, of tumor tissue. 


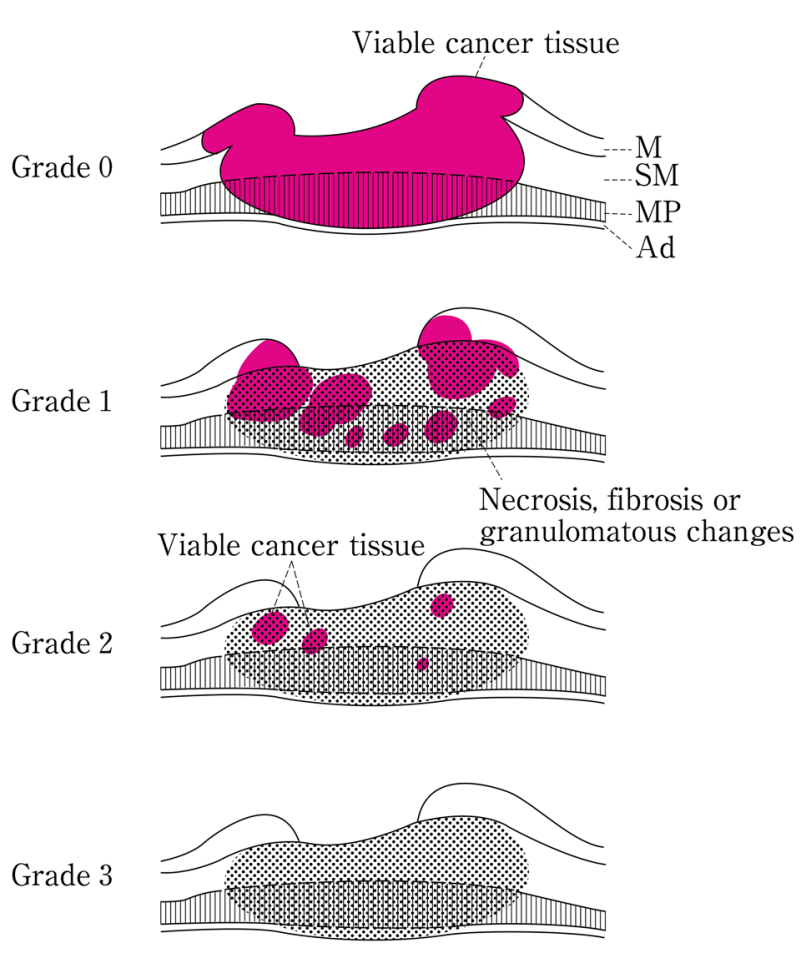

Fig. 1-16 Histological efficacy of chemotherapy and/or radiotherapy

Grade 2: Moderately effective

Viable cancer cells account for less than $1 / 3$ of tumor tissue, while other cancer cells are severely degenerated or necrotic.

Grade 3: Markedly effective

No viable cancer cells are evident.

Note: Definite re-proliferation of tumor cells in treated cancer lesions, after preoperative treatment, should be recorded as "re-proliferation $(+)$ ".

\subsection{Lymph node metastasis $(\mathrm{pN})$}

Note 1: Lymph nodes should be sectioned through the hilum.

Note 2: The number of dissected lymph nodes and metastatic lymph nodes should be recorded.

Note 3: The metastatic ratio (the number of metastatic lymph nodes/the number of dissected lymph nodes) is described for each lymph node station. The total metastatic ratio is also described in parentheses.

e.g.: No.104R (0/10), No.104 L (1/13), No.106recR (1/ $3)$, No.106recL (0/4).

Note 4: Metastasis to soft tissue without a lymph node structure is described as extra-lymph node metastasis, and the locations and number of metastases are recorded. e.g.: lymph node metastasis, $1 / 25$; extra-lymph node metastasis, $2 / 2$.

Note 5: Extranodal invasion including direct invasion and lymphatic invasion is described.

Note 6: A lymph node with no viable cancer cells after preoperative treatment is diagnosed as negative for metastasis (pN0).

[Reference]

Japanese Gastric Cancer Association: Japanese Classification of Gastric Carcinoma (in Japanese). 13th ed. Kanehara Shuppan, Tokyo, 1999; 27.

4.4. Distant organ metastasis (pM) (cf. 3.5 Distant organ metastasis (M))

4.5. Residual tumor (pR) (cf. 3.6 Residual tumor (R))

4.6. Curativity (pCur) (cf. Curativity (Cur))

\section{Endoscopic treatment}

5.1. Handling of specimens resected endoscopically ${ }^{\text {Note }}$

Extending fixation of the resected specimen: A specimen is extended and fixed immediately after resection on a cork board or polystyrene foam and is fixed in formalin solution of sufficient volume for at least half a day.

Note: As for the extended fixation of the resected specimen, it should be done by the doctor or co-worker who carried out endoscopic treatment. Especially in piecemeal resection, fixation of the specimen should be performed by the doctor(s) aware of the actual figure of the tumor in vivo to enable more exact restructuring.

5.2. Description of macroscopic findings and endoscopic findings

An e-mark is prefixed to macroscopic findings and endoscopic findings.

\subsubsection{Number of tumors and number of resected specimens}

Number of lesions

Number of specimens resected from each tumor (number of specimens):

1. en bloc resection, 2. piecemeal resection.

\subsubsection{Size of resected specimen and size of tumor lesion (for each lesion)}

The size is described by the greatest longitudinal dimension in millimeters multiplied by the greatest transverse dimension in millimeters: $\mathrm{a} \times \mathrm{b}(\mathrm{mm})$. 


\subsubsection{Tumor types}

The tumor types are classified into Type 0-I, Type 0-IIa, Type 0-IIb, Type 0-IIc, Type 0-III, combined type, and others.

\subsubsection{Macroscopic findings}

\subsubsection{Horizontal margin (eHM)}

eHMX Whether residual tumor is present on the horizontal margin cannot be assessed.

eHM0 Non-cancerous squamous epithelium and lamina propria mucosae have been confirmed on all horizontal resection margins.

eHM1 The tumor is exposed on one of its horizontal resection margins.

\subsubsection{Vertical margin (eVM)}

eVMX Whether residual tumor is present on the vertical margin cannot be assessed.

eVM0 The tumor is not exposed on any of its vertical margins.

eVM1 The tumor is exposed on one of its vertical margins.

\subsubsection{Clinical assessment of residual tumor ${ }^{\text {Note } 1,2}$}

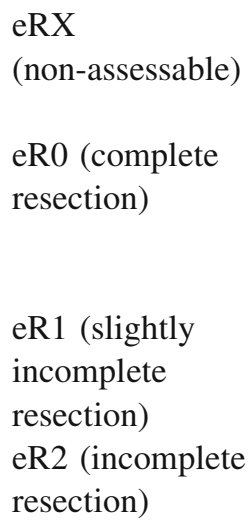

Whether residual tumor is present on the resection margin cannot be assessed.

Non-cancerous squamous epithelium and lamina propria mucosa have been confirmed on all resection margins.

Presence of an iodine-unstained area on the margin of the resected specimen.

Presence of residual tumor.

Note 1: The clinical assessment of the residual tumor, referring to the iodine staining of the resected specimen, should be performed immediately after the endoscopic resection. In cases with a piecemeal resection, iodine staining of the ulcer margin after resection should be referenced.
Note 2: This assessment method should be applied to squamous cell carcinoma.

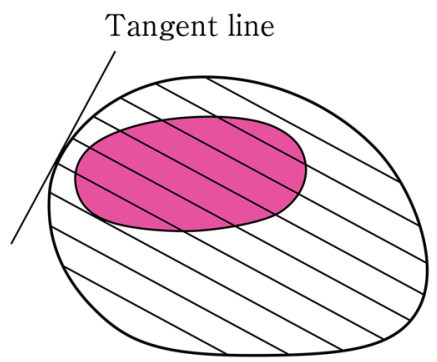

Fig. 1-17 How to cut endoscopically resected specimens

\subsection{Preparation for pathological examination (Fig. 1-17)}

Before cutting, formalin-fixed specimen should be stained with iodine solution to confirm unstained area. ${ }^{\text {Note }}$ Cutting lines are decided as crossing lines at right angles to the tangent line at the resection margin closest to the tumor, and a whole resected specimen is cut in slice each $2-3 \mathrm{~mm}$ thick.

Note: Rinsing the sample with tap water for at least 30 min can result in a good staining condition. To make a clearer contrast between stained and unstained areas, the sample should be treated with relatively low concentration $(0.1-0.5 \%)$ of iodine solution for a little bit longer time.

\subsection{Description of pathological findings}

The pathological diagnosis of an endoscopically resected specimen is summarized by the histological type, depth of tumor invasion, assessment of resection margin (horizontal and vertical), and vascular invasion.

\subsubsection{Pathological diagnosis}

The diagnosis is based on the histological classification (4.2.1). Although most esophageal tumors are squamous cell carcinoma, the evaluation of histological differentiation is omitted for intraepithelial carcinoma.

\subsubsection{Depth of tumor invasion ( $p T)$}

A mucosal cancer is categorized in three depths of pT1aEP, pT1a-LPM and pT1a-MM. In a submucosal cancer, the 
distance from the lamina muscularis mucosae is described because the entire submucosal layer cannot be examined in an endoscopically resected specimen. A submucosal cancer is sub-classified as pT1b-SM1 (submucosal tumor invasion limited to within $200 \mu \mathrm{m}$ ) and pT1b-SM2 (invasion to more than $200 \mu \mathrm{m}$ ).

e.g.: pT1b-SM2, $300 \mu \mathrm{m}$.

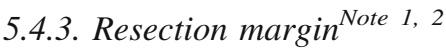

\subsubsection{Horizontal margin (pHM)}

pHMX It cannot be assessed whether there is residual tumor on the horizontal margin or not.

pHM0 Non-cancerous squamous epithelium and lamina propria mucosae are confirmed on all horizontal resection margins.

pHM1 The tumor is exposed on any horizontal resection margin.

\subsubsection{Vertical margin (pVM)}

pVMX It cannot be assessed whether there is residual tumor on the vertical margin or not.

pVM0 No tumor is exposed on any vertical margin.

pVM1 The tumor is exposed on any vertical margin.

Note 1: When no tumor is recognized in any resection margin, it is defined as a complete resection (pR0), and when a tumor is recognized in any resection margin, it is defined as an incomplete resection (pR1).

Note 2: When vascular invasion is present in the resection margin, it is defined as a positive resection margin (pHM1, pVM1).

\subsubsection{Non-assessable resection margin (pRX)}

1. Because of crushing injury or the burn effect in the specimen during endoscopic resection, non-cancerous tissue in the resection margin cannot be confirmed.

2. Reconstruction after piecemeal resection is impossible. ${ }^{\text {Note }}$

3. Suspected residual tumor in the basal layer because of non-continuous tumor extension.

4. Possible residual tumor in the vertical margin because of intra-ductal spread.

5. Indeterminable residual tumor because of other reasons.

Note: In piecemeal resection $\mathrm{pR} 0$ is confirmed only when restructuring is possible and only non-cancerous tissue is recognized at the resection margins of the restructured specimen.

\subsubsection{Infiltrative growth pattern (INF)}

INFa (expansive Expansive growth of tumor nests with a type) well-demarcated border from the surrounding tissue.

$\mathrm{INFb} \quad$ Intermediate growth pattern, between

(intermediate INFa and INFc.

type)

INFc

(infiltrative

type)

Infiltrative growth of tumor nests with an ill-defined border from the surrounding tissue.

\subsubsection{Vascular invasion $(l y / v)^{\text {Note }}$}

It is not necessary to evaluate the degree of vascular involvement: only its presence or absence should be described.

\subsubsection{Lymphatic invasion (ly)}

ly (-) No lymphatic invasion.

ly (+) Lymphatic invasion

5.4.5.2. Venous invasion (v)

$\mathrm{v}(-)$ No venous invasion

$\mathrm{v}(+)$ Venous invasion

Note: A special staining method for elastic fibers of the vascular wall, such as Elastica van Gieson (EVG) or Victoria blue (VB) staining, is needed to determine venous invasion. Immunostaining with an anti-D2-40 antibody is useful to confirm lymphatic invasion. When differentiating between lymphatic and venous invasion is difficult, the case should be described as ly/v. When lymphatic and/or venous invasion is prominent, this evaluation should be included in addition to ly $(+)$ or v $(+)$.

\subsubsection{Report of pathological findings}

All the above-mentioned factors should be described, and the attachment of a figure showing the general view of the resected specimen with the regional depths of tumor invasion and vascular invasion is recommended. It is better to attach a schematic figure showing pathological findings on the cut surface if necessary.

\subsection{Residual tumor $(\mathrm{pR})^{\text {Note } 1,2}$}

pRX The existence of residual tumor at the resection margin cannot be assessed pathologically. 
pR0 No cancer tissue is pathologically present at any margin of the resected specimen.

pR1 Cancer tissue is pathologically present at the margin of the resected specimen.

pR2 A cancer lesion is present.

Note 1: For a piecemeal resection, the presence of residual tumor is evaluated after rebuilding the specimen.

Note 2: When multiple lesions are resected, each lesion is evaluated individually.

\subsection{Curativity (pCur)}

When endoscopic resection (EMR: endoscopic mucosal resection, or ESD: endoscopic submucosal dissection) is performed for superficial esophageal cancer that does not exhibit clinical metastasis, a comprehensive evaluation can be established, based on the pathological findings of depth of invasion, residual tumor, and vascular invasion (Table 1-9).

\begin{tabular}{|c|c|}
\hline $\begin{array}{l}\text { Curativity A } \\
\text { (pCur A) }\end{array}$ & pT1a-EP or pT1a-LPM with pR0. \\
\hline $\begin{array}{l}\text { Curativity B } \\
\text { (pCur B) }\end{array}$ & $\begin{array}{l}\text { pT1a-EP or pT1a-LPM with pRX. } \\
\text { pT1a-MM or pT1b-SM1 with pR0 or } \\
\text { pRX. }\end{array}$ \\
\hline $\begin{array}{l}\text { Curativity C } \\
\text { (pCur C) }\end{array}$ & $\begin{array}{l}\text { pT1b-SM2, positive micro vascular } \\
\text { permeation despite depth of invasion, } \\
\text { pR1 or pR2. }\end{array}$ \\
\hline
\end{tabular}

Table 1-9 Curativity of the endoscopic resection

\begin{tabular}{llll}
\hline Depth of tumor invasion (T) & $\begin{array}{l}\text { Residual tumor } \\
(\mathrm{R})\end{array}$ & Others \\
\cline { 2 - 3 } & pR0 & $\mathrm{pRX}$ & $\begin{array}{l}\mathrm{pR} 1, \mathrm{pR} 2 \text { and/or } \\
\mathrm{v}+, \text { ly+ }\end{array}$ \\
\hline pT1a-EP & $\mathrm{A}$ & $\mathrm{B}$ & $\mathrm{C}$ \\
pT1a-LPM & $\mathrm{A}$ & $\mathrm{B}$ & $\mathrm{C}$ \\
pT1a-MM & B & B & $\mathrm{C}$ \\
pT1b-SM1 & B & B & $\mathrm{C}$ \\
pT1b-SM2 & C & C & C \\
\hline
\end{tabular}

\section{Barrett esophagus and adenocarcinoma in Barrett esophagus}

6.1. Definition and description methods for Barrett mucosa, Barrett esophagus and Adenocarcinoma in Barrett esophagus

\subsubsection{Definition of the esophagogastric junction (EGJ)}

The EGJ should be defined systematically in accordance with the criteria listed below. Among these criteria, endoscopic findings should be given priority over findings obtained using other diagnostic modalities.

1. Endoscopic findings

Lower margin of palisading small vessels

If the palisading small vessels are unclear, the oral margin of the longitudinal folds of the greater curvature of the stomach is defined as the EGJ.

2. Upper gastrointestinal series (UGI)

Narrowest locus of the lower esophagus

In the presence of a sliding hiatal hernia, the upper end of the longitudinal folds is defined as the EGJ.

In the presence of Barrett esophagus, the upper end of the longitudinal folds is defined as the EGJ.

3. Pathological study

Macroscopic definition: The EGJ should be defined macroscopically as the point at which the luminal caliber changes in the area where the tubular esophagus is connected to the vestibule lumen of the stomach.

Microscopic definition: For a mucosal layer with intact structures, the EGJ should be defined as follows:

1. Non-Barrett esophagus: The EGJ is defined as the squamocolumnar junction.

2. Barrett esophagus: Histological structures such as proper esophageal glands and their ducts, a double-layer muscularis mucosae, or palisading small vessels should be included in the microscopic definition of the EGJ.

For a non-intact mucosal layer, the EGJ should be defined based on the macroscopic findings of the surgical specimen, and the EGJ should be presumed based on the presence of histological structures associated with the esophagus or stomach.

\subsubsection{Barrett mucosa}

Columnar epithelium continuous from the stomach with or without intestinal metaplasia

\subsubsection{Barrett esophagus}

An esophagus containing Barrett mucosa should be designated as Barrett esophagus. Note 1

At least one of the following conditions must be satisfied.

1. Presence of esophageal gland ducts in the mucosal layer or proper esophageal glands in the submucosal layer within the area of columnar epithelium.

2. Presence of squamous islands in the columnar epithelium. 
3. Presence of a double-layer muscularis mucosae $e^{\text {Notes } 2,3}$

Note 1: The presence of circular Barrett mucosa extending longitudinally for $3 \mathrm{~cm}$ or more is called long segment Barrett esophagus (LSBE) (Fig. 1-18). On the other hand, the presence of circular Barrett mucosa less than $3 \mathrm{~cm}$ in length or the presence of non-circular Barrett mucosa is designated as short segment Barrett esophagus (SSBE) (Fig. 1-19).

Note 2: New muscularis mucosae can sometimes be found immediately under the columnar epithelium. In the Japanese Classification, the primary muscularis mucosae is called deep muscularis mucosae (DMM), and the new muscularis mucosae is called superficial muscularis mucosae (SMM). The identification of SMM and DMM is occasionally difficult because of the fusion of both layers, the thickness of the layer, or the presence of irregularities.

Note 3: Barrett esophagus can also be defined as the presence of columnar epithelium containing small palisading vessels with diameters of greater than $100 \mu \mathrm{m}$ within the lamina propria mucosae.

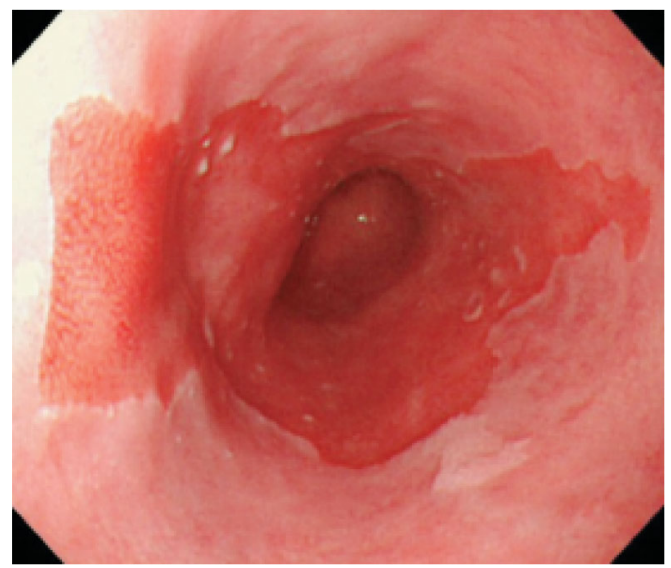

Fig. 1-18 Long segment Barrett's esophagus (LSBE)

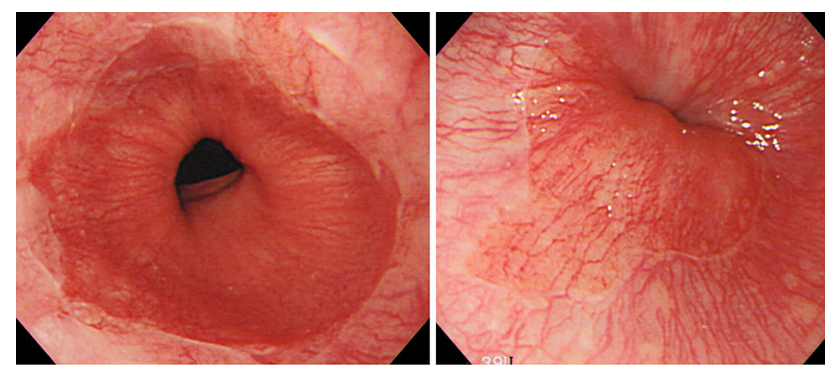

Fig. 1-19 Short segment Barrett esophagus (SSBE). Circular Barrett mucosa extending for less than $3 \mathrm{~cm}$ in length or non-circular Barrett mucosa is designated as short segment Barrett esophagus (SSBE). a Circular SSBE. b Non-circular SSBE

\subsubsection{Adenocarcinoma in Barrett esophagus}

Adenocarcinoma arising in Barrett mucosa ${ }^{\text {Note } 1}$

Note 1: The origin of adenocarcinoma in areas of Barrett esophagus is not the same as that of gastric adenocarcinoma, which is derived from gastric mucosa adjacent to the EGJ. Gastric cancer is described according to the Japanese Classification of Gastric Cancer, and esophageal adenocarcinoma is described according to the Japanese Classification of Esophageal Cancer. However, the histological classifications of both adenocarcinomas are described according to the Japanese Classification of Gastric Cancer.

Circular Barrett mucosa extending longitudinally for $3 \mathrm{~cm}$ or more is called long segment Barrett esophagus (LSBE).

\subsection{Tumor location}

Same as that for esophageal cancer.

Note: In cases with a hiatus hernia, the tumor location should be determined using barium contrast radiography.

\subsection{Description of tumors}

Adenocarcinoma in Barrett esophagus is described according to the Japanese Classification for Esophageal Cancer, except for the depth of tumor invasion.

\subsubsection{Primary tumor}

\subsubsection{Circumferential location}

\subsubsection{Tumor size}

\subsubsection{Macroscopic tumor types}

\subsubsection{Depth of tumor invasion (T)}

TX Depth of tumor invasion cannot be assessed.

T0 No evidence of primary tumor.

T1a Tumor has invaded the mucosa.

T1a-SMM Tumor has invaded the superficial muscularis mucosae (SMM).

T1a-LPM Tumor has invaded the lamina propria mucosae.

T1a-DMM Tumor has invaded the deep muscularis mucosae (DMM).

T1b Tumor has invaded the submucosa (SM).

SM1 Tumor has invaded the upper third of the submucosa. 
SM2 Tumor has invaded the middle third of the submucosa.

SM3 Tumor has invaded the lower third of the submucosa.

T2 Tumor has invaded the muscular propria.

T3 Tumor has invaded the adventitia.

T4 Tumor has invaded adjacent structure(s).

\subsubsection{Intramural metastasis (IM)}

Same as that for esophageal cancer.

\subsubsection{Lymph node metastasis $(N)^{\text {Note }}$}

Note: In cases with a hiatal hernia, the field of lymph node dissection should be designated according to the tumor location as defined using Barium contrast radiography.

\subsubsection{Distant organ metastasis (M)}

Same as that for esophageal cancer.

\subsection{Stage}

Same as that for esophageal cancer.

\section{Treatment}

\subsection{Endoscopic treatment}

\subsubsection{Endoscopic resection: $E R$}

7.1.1.1. Endoscopic mucosal resection: EMR

\subsubsection{Endoscopic submucosal dissection: ESD}

Note: The following items should be described.

Method of resection: (1) en bloc resection, (2) piecemeal resection.

Residual tumor: eRX, eR0, eR1, eR2.

Complications: (1) perforation, (2) bleeding requiring treatment, (3) stenosis, (4) others (including mediastinal emphysema).

Combined therapy: (1) none, (2) APC, (3) laser, (4) PDT, (5) MCT, (6) others.

\subsubsection{Other Endoscopic treatments}

7.1.2.1. Argon plasma coagulation: APC

7.1.2.2. Laser therapy: laser
7.1.2.3. Photodynamic therapy: PDT

7.1.2.4. Microwave coagulation therapy: MCT

7.1.2.5. Others

Note: Any other therapy performed should be described.

7.2. Surgical treatments

\subsubsection{Resection and reconstruction procedures}

7.2.1.1. Staged operations

One-stage operation

Staged operation

\subsubsection{Surgery with multi-modality treatments}

Planned surgery: planned surgery after neoadjuvant chemotherapy, radiotherapy, or both.

Salvage surgery: surgery for cases with residual tumor or cases with recurrent tumor after definitive chemoradiotherapy with more than 50 Gy radiation.

The surgical methods, such as esophagectomy, lymphadenectomy, endoscopic resection, and so on, should be described.

\subsubsection{Approaches for tumor resection}

\section{Endoscopic}

Thoracoscopic, thoracoscopy-assisted

Mediastinoscopic

Laparoscopic, laparoscopy-assisted
Transcervical
Thoracotomy
Right
Left
Laparotomy
Thoracoabdominal incision
Right
Left
Transhiatal
Sternotomy

Note: When several approaches are adopted, only the main approach should be described.

\subsubsection{Extent of esophageal resection}

Total esophagectomy: the cervical, thoracic and abdominal esophagus is resected, regardless of whether laryngectomy is done or not. 
Subtotal esophagectomy: almost all the thoracic esophagus is resected

Middle and lower esophagectomy: the middle and lower esophagus (including abdominal esophagus) is resected Note 1

Lower esophagectomy: the lower esophagus (including abdominal esophagus) is resected

Partial esophagectomy: resection of full-thickness partial esophagus $^{\text {Note } 2}$

Mucosal resection: resection of mucosal and submucosal layers

Others

Note 1: Lower esophagectomy includes resection of the lower esophagus and cardia.

Note 2: In partial esophagectomy, the location of the esophagus resected should be described.

\subsubsection{Combined resection}

The organ(s) resected together because of cancer invasion should be described.

Note: Total or partial resection of the organ(s) should be described.

\subsubsection{Reconstruction}

\subsection{Reconstruction routes}

Antethoracic (subcutaneous)

Retrosternal

Posterior mediastinal

\subsection{Sites of anastomosis}

Neck

Antethoracic (subcutaneous)

Thoracic cavity (proximal, distal)

Lower mediastinum

Note: The border between the proximal and the distal site of anastomosis is the upper level of the aortic arch.

\subsection{Organs used for reconstruction}

\section{Stomach}

Whole stomach

Gastric tube

Jejunum

Pedicled

Free

Colon

$$
\text { Pedicled }^{\text {Note } 1}
$$

Left colon ${ }^{\text {Note } 2}$

Right colon ${ }^{\text {Note } 3}$

Ileum and colon

Free

Colon

Ileum and colon

Skin and muscle ${ }^{\text {Note } 4}$

Skin flap

Local skin flap

Pedicled

Free

Musculocutaneous flap

Pedicled

Free

Note 1: Isoperistaltic or antiperistaltic should be described.

Note 2: For reconstruction with left colon, the transverse colon with left colic artery is used.

Note 3: For reconstruction with right colon, the ascending colon with the middle colic artery is used.

Note 4: The name of the skin flap or muscle flap that is used should be described.

e.g.: free forearm skin flap, right pectoralis major musculocutaneous flap.

\subsubsection{Conservative/palliative procedures}

\subsubsection{Stoma}

Pharyngostomy

Esophagostomy

Gastrostomy

Jejunostomy

\subsubsection{Bypass}

Pharyngogastrostomy

Esophagogastrostomy

Esophagojejunostomy

Esophagocolostomy

7.2.2.3. Exploratory thoracotomy, exploratory laparotomy

7.2.2.4. Others

Lymph node dissection without esophagectomy 


\subsection{Stenting}

\subsubsection{Esophageal stents}

(a) Therapy before stenting

No

Yes (chemotherapy, radiotherapy, chemoradiotherapy, others)

(b) Therapy after stenting

No

Yes (chemotherapy, radiotherapy, chemoradiotherapy, others)

(c) Esophageal fistula

No

Yes

(d) Type of stent

Covered or non-covered

Anti-reflux valve (yes, no)

Diameter

Length

(e) Operative complications

No

Yes (bleeding requiring treatment, dyspnea, pain, others)

(f) Evaluation of stent

Status of eating before stenting

Status of eating at discharge

(g) Complications after stenting

No

Yes (bleeding requiring treatment, perforation, pain, regurgitation of gastric contents, dyspnea, migration, esophago-airway fistula, others)

\subsubsection{Tracheobronchial stents}

Type of stent.

\subsubsection{Aortic stents}

Type of stent.

7.4. Common issues for radiotherapy and chemotherapy

\subsubsection{Disease status}

Untreated

Macroscopic residual after surgery

Microscopically residual after surgery

No obvious residual disease after surgery

Loco-regional recurrence after surgery

Distant metastasis after surgery
Other postoperative recurrence

Residual disease after EMR/ESD

No obvious residual disease after EMR/ESD

After stenting

After intraoperative radiation therapy for macroscopic disease

After intraoperative radiation therapy for no obvious macroscopic disease

\subsubsection{Aim of treatment}

Definitive

Palliative

Preoperative

Preventive

Recurrent disease

Others

\subsubsection{Reasons for definitive radiotherapy}

Severe associated disease(s)

Advanced age

Patient's wishes

Others

7.5. Radiotherapy (RT)

\subsubsection{Clinical target volume (CTV)}

Primary lesion

Entire esophagus

Resected lymph node area for prevention (supra-clavicular, mediastinal, abdominal)

Distant organ metastasis

\subsubsection{Methods of radiotherapy}

External beam radiation therapy

External beam radiation therapy + intra-cavitary radiation therapy

Intra-cavitary radiation therapy alone

\subsubsection{External beam radiotherapy}

X-ray

Proton

Carbon

Gamma-ray

Electron

7.5.3.1. Planning methods

X-ray simulator

Three dimensional 


\subsubsection{Field setting}

Opposing

Anterior oblique

Three directions

More than 4 fields

Rotating

Intensity-modulated radiotherapy (IMRT)

\subsubsection{Reference points}

CTV center

Field center

Others

\subsubsection{Dose calculation}

Non-homogeneity correction

Algorithm

7.5.3.5. Dose fractionation of external beam radiotherapy

Dose/fraction

Number of fractions/week

Total dose

Overall treatment time

\subsubsection{Intraluminal irradiation}

Low dose rate $\left({ }^{226} \mathrm{Ra}\right)$

High dose rate $\left({ }^{192} \mathrm{Ir},{ }^{60} \mathrm{Co},{ }^{137} \mathrm{Cs}\right)$

\subsubsection{Reference points}

Mucosal surface mm below mucosa

\subsubsection{Dose fractionation of intraluminal irradiation}

Dose/fraction

Number of fractions/week

Total dose

Overall treatment time

\subsubsection{Completion of treatment}

Complete without break

Complete with break

Incomplete

\subsubsection{Reasons for treatment cessation}

Completion of the planned treatment

Disease progression
Adverse events

Patient's refusal (related to adverse events)

Patient's refusal (not related to adverse events)

7.6. Chemotherapy (CT)

7.6.1. Agents

Name of agent (generic name should be recorded).

\subsubsection{Administration routes}

Intravenous

Oral

Transarterial

Local injection (including abdominal and chest cavity)

Others

\subsubsection{Administration procedures}

Bolus

Continuous

Others

\subsubsection{Administration doses}

Dose should be recorded as per body surface area $\left(/ \mathrm{m}^{2}\right)$ or per body (/body).

\subsubsection{Administration schedules}

Course duration

Course interval

Upper limit in number of courses.

\subsubsection{Duration of administration}

Initial date of administration

Last date of administration

Total number of courses

\subsubsection{Total administration dose}

Total administration dose of each agent should be calculated as per body surface area or per body.

\subsubsection{Reasons for treatment cessation}

Completion of the planned treatment

Disease progression

Adverse events

Patient's refusal (related to adverse events)

Patient's refusal (not related to adverse events)

Others 


\subsubsection{Adverse events}

Recorded in accordance with the "Common Terminology Criteria for Adverse Events ver. 4, (Japanese version) JCOG/JSCO edition".

\subsection{Multi-modality treatment}

7.7.1 Combination of endoscopic treatment and surgery, radiotherapy, chemoradiotherapy or chemotherapy

\section{Classification Preoperative, intraoperative, or postoperative. \\ Description Planned treatment or salvage treatment Surgery Evaluation of residual tumor (pR) (If a residual tumor is present, describe the tumor location, depth of tumor invasion, tumor margin, presence of vascular invasion, presence of lymph node metastasis, etc.) \\ Multiple tumors: yes or no (If multiple tumors are present, describe the tumor location and the number of lesions.)}

Note: Evaluate residual tumors of primary and metastatic lesions.

Radiotherapy: radiation field, total dose

Chemotherapy: regimen

\subsubsection{Chemoradiotherapy (CRT)}

Classification according to timing: concurrent or sequential Classification according to intent: definitive, neoadjuvant, or after surgery (adjuvant, additive)

Note: R0 resection should be recorded as adjuvant. R2 should be recorded as additive.

\subsection{Hyperthermia (HT)}

7.9. Immunotherapy (IT)

\section{Results of treatment}

The following matters are recorded to allow precise statistical analysis for a comprehensive registry of esophageal cancer.

\subsection{Total number of patients}

Total number of outpatients

Total number of admitted patients

Total number of patients admitted for various treatments

8.2. Multiple primary cancers

Primary lesion of another cancer, diagnosis (clinical and pathological), synchronous or metachronous, and treatment

8.3. Main treatment and adjuvant therapy

Endoscopic treatment

Surgical treatment

Palliative operation

Radiotherapy

Chemotherapy

Chemoradiotherapy

Other non-surgical treatment

No treatment

8.4. Total number of patients treated, and number and rate of patients treated with each procedure

\subsubsection{Patients operated}

The total number of patients admitted, the number of patients who underwent resection, the numbers of patients with curative and non-curative resections, and the resection rate should be recorded.

Resection rate $=$ patients who underwent resection/patients admitted ${ }^{\text {Note } 1}$

Total number and ratio of patients with or without curative resection $^{\text {Notes 2, } 3}$

Note 1: Patients who have undergone an esophagectomy for the first time are evaluated.

Note 2: Curative operation is defined as Curativity A or $\mathrm{B}$ resection.

Note 3: Non-curative operation is defined as Curativity $\mathrm{C}$ resection.

\subsubsection{Patients with Endoscopic treatment}

Record the number of patients who underwent endoscopic treatment, and the total number and ratio of patients with or without curative resection.

Cases of treatment completed only by endoscopic treatment are recorded separately from operated cases as endoscopically treated cases. 
One-piece resection rate $=$ (number of patients who underwent one-piece resection/total number of patients who underwent endoscopic resection) $\times 100^{\text {Note }}$

Curativity rate $=$ (number of patients who underwent curative endoscopic resection/total number of patients who underwent endoscopic resection) $\times 100$

Note: The number of patients who underwent endoscopic resection indicates those who underwent endoscopic mucosal dissection and endoscopic submucosal dissection. It does not include the number of patients treated by laser therapy or photodynamic therapy.

\subsubsection{Patients with chemotherapy and/or radiotherapy}

Total numbers and rates according to response evaluation criteria after treatment.

\subsection{Operative mortality ${ }^{\text {Note }}$}

Operative mortality $=$ (operative deaths/patients operated) $\times 100$

Mortality after esophagectomy $=$ (deaths after esophagectomy/patients who underwent esophagectomy) $\times 100$

Note: Operative death means death within 30 days after operation in or out of hospital.

8.6. Hospital mortality ${ }^{\text {Notes } 1,2}$

Hospital mortality $=($ hospital deaths/patients operated $) \times$ 100

Hospital mortality after esophagectomy $=$ (hospital deaths after esophagectomy/patients who underwent esophagectomy) $\times 100$

Note: Hospital death is defined as death during the same hospitalization, regardless of department at time of death.

\subsection{Long-term outcome}

The following items should be recorded for survival analysis.

\subsubsection{Alive or dead}

Alive: The date of the most recent follow-up

Death: The date of death

Unknown: The date of the most recent follow-up

Cause of death

Treatment-related death: death because of surgical treatment, chemotherapy, radiotherapy, or other therapies
Death because of esophageal cancer

Death because of another cancer: primary cancerrelated deaths should be recorded.

Death because of another disease: the name(s) of the disease(s) should be recorded.

Death because of accident: suicide should be included. Death because of unknown cause(s): this category should be basically regarded as esophageal cancerrelated deaths.

\subsubsection{Recurrence}

Yes or No

Date of recurrence

Pattern and site of recurrence: Each recurrence should be recorded chronologically

Local recurrence

Primary lesion (esophagus) $)^{\text {Note } 1}$

Recurrence in the mediastinum adjacent to the primary lesion

Recurrence at the anastomotic site or in the esophageal stump

Recurrence in the regional lymph nodes

Others including intramural metastasis in the esophagus or stomach

Distant metastasis ${ }^{\text {Note } 2}$

Lymphogenous recurrence

Hematogenous recurrence (distant organ(s))

Disseminated recurrence (pleura, peritoneum, pericardium)

Unknown

Note 1: Recurrence at the same place as the primary lesion in the esophagus can occur after esophaguspreserving treatment including endoscopic mucosal resection, chemotherapy and radiotherapy.

Note 2: Recurrent organ(s) are indicated by the abbreviations of the TNM classification.

Liver: HEP, Lung: PUL, Peritoneum: PER, Lymph node: LYM, Bone: OSS.

Brain: BRA, Kidney: REN, Adrenal gland: ADR, Skin: SKI, Others: OTH.

8.8. Long-term outcomes and prognosis, especially survival rate

\subsubsection{Analysis of survival rates}

Target (operation, endoscopic treatment, curativity and so on) 
The calculation method of survival rates

Crude survival rate: direct method, cumulative method (Life-table method, Kaplan-Meier method)

Relative survival rate

Censored cases: overall survival rate, cause-specific survival rate

Statistical analysis of survival rate

Rate of cases lost to follow-up

\subsubsection{Period and rate of esophageal preservation}

This is the period during which the esophagus is preserved in patients with esophageal cancer who underwent nonesophagectomy treatment such as endoscopic mucosal resection, endoscopic submucosal dissection, chemotherapy and/or radiotherapy. The rate of those patients per all patients who underwent treatment for esophageal cancer is described.

\section{[Reference]}

Murakami M, Kuroda Y, Matsumoto S, et al. Treatment results of esophageal carcinoma of clinical T3, T4 M0: histological comparison between neoadjuvant chemoradiotherapy followed by surgery or definitive radiotherapy and conventional surgery. Oncol Rep 2000; 7: 571-578.

\subsection{Terminology related to survival period}

\subsubsection{Survival time}

Time until death from the time of a certain event

\subsubsection{Overall survival (OS)}

Time until death regardless of cause from the initial date. In observation studies, the day starting treatment, or the day confirming diagnosis is used as the initial date. In clinical trials, the "registration date" (the allocation day in the case of a randomized controlled trial) is used.

\subsubsection{Median survival time (MST)}

Period from the initial date to the first date of the survival rate being less than $50 \%$ in the survival curve calculated by the Kaplan-Meier method. The initial date is the same as described in 8.9.2.

\subsubsection{Survival rate}

The rate of survivors at a given time

8.9.5. Progression-free survival (PFS), time to progression (TTP)

It is the shortest period among periods from the initial date to progression or recurrence, or death. Death from any cause is regarded as an event, in PFS. Death is only regarded as an event if it is caused by esophageal cancer, in TTP.

8.9.6. Relapse-free survival, recurrence-free survival (RFS)

This is the period between the initial date to recurrence or death.

The day when the disease-free state was achieved such as an operation day, is used as the initial date.

\subsubsection{Disease-free survival (DFS)}

It is the shortest period among the periods from the initial date to a recurrence, death, or diagnosis of a second primary cancer. The day when the disease-free state such as an operation day was achieved, is used as the initial date.

\subsubsection{Time to treatment failure (TTF)}

This is the shortest period among the periods from the initial date to treatment cessation, progression, and death.

\subsubsection{Response duration}

Period from the first day when the disease was assessed as CR or PR until progression.

\subsubsection{Complete response duration}

Period from the first day when the disease was assessed as CR until recurrence.

[Reference]

Japan Society for Clinical Oncology. Terminology in Clinical Oncology 2013 (in Japanese)

Open Access This article is distributed under the terms of the Creative Commons Attribution 4.0 International License (http://crea tivecommons.org/licenses/by/4.0/), which permits unrestricted use, distribution, and reproduction in any medium, provided you give appropriate credit to the original author(s) and the source, provide a link to the Creative Commons license, and indicate if changes were made. 